\title{
Advanced strategies for therapeutic targeting of wild-type and mutant
}

\section{p53 in cancer}

Shengliang Zhang ${ }^{1-4}$, Lindsey Carlsen ${ }^{1,5}$, Liz Hernandez Borrero ${ }^{1}$, Attila A. Seyhan ${ }^{1-4}$, Xiaobing Tian ${ }^{1-4}$, and Wafik S. El-Deiry ${ }^{1-4,6^{*}}$

1.Laboratory of Translational Oncology and Experimental Cancer Therapeutics, Warren Alpert Medical School, Brown University, Providence, Rhode Island, USA

2.Department of Pathology and Laboratory Medicine, Warren Alpert Medical School, Brown University, Providence, Rhode Island, USA

3.Joint Program in Cancer Biology, Lifespan Health System and Brown University, Providence, Rhode Island, USA

4.Legorreta Cancer Center at Brown University, Providence, Rhode Island, USA

5.Pathobiology Graduate Program, Warren Alpert Medical School, Brown University, Providence, Rhode Island, USA

6. Hematology/Oncology Division, Department of Medicine, Lifespan Health System and Brown University, Providence, Rhode Island, USA

Running title: Cancer therapy of targeting mutant and wild p53

Key words: P53; mutant p53; targeting therapy; immunotherapy; cancer

*Correspondence: wafik@brown.edu 


\section{Abstract}

TP53 is a tumor suppressor gene that encodes a sequence-specific DNA-binding transcription factor activated by stressful stimuli and upregulates target genes involved in growth suppression, cell death, DNA repair, metabolism, among others. P53 is the most frequently mutated gene in tumors with mutations not only leading to loss-of-function (LOF), but also gain-of-function (GOF) which promotes tumor progression, and metastasis. The tumor-specific status of mutant p53 protein has suggested it is a promising target for cancer therapy. We summarize the current progress of targeting wildtype and mutant p53 for cancer therapy through biotherapeutic and biopharmaceutical methods for 1) boosting p53 activity in cancer, 2) p53-dependent and p53-independent strategies for targeting p53 pathway functional restoration in p53-mutated cancer, 3) targeting p53 in immunotherapy, and 4) combination therapies targeting p53, p53 checkpoints, or mutant p53 for cancer therapy. 


\section{Introduction}

p53 protein is a sequence-specific DNA-binding transcription factor regulating gene expression related to multiple cellular functions including, but not limited to, cell cycle arrest, cell apoptosis, cell growth, DNA repair, cell metabolism, and the immune response in response to stressful stimuli [1]. Clinical studies show that wild-type p53 sensitizes cancer cells to conventional chemoradiotherapy for example in treatment of rectal cancer. Nearly half of tumors harbor mutant p53. Most p53 mutations are single missense mutations in its functional domains, most commonly the central DNA-binding domain. Missense mutations not only cause mutant p53 protein to lose its wild-type functions (LOF) and acquire dominant-negative activities, but also endow mutant p53 with gain-of-function (GOF) capabilities that confer aggressive tumor behavior and drug resistance. Clinical studies correlate poor prognosis of cancer patients with mutant p53. p53 is considered as a biomarker for tumor progression and an excellent (yet relatively unexploited) target for designing cancer therapeutic strategies. Even in cancers carrying wild-type p53, aberrant p53 pathway signaling often occurs due to abnormal regulation such as due to high MDM2 expression often from gene amplification. The different p53 status between cancer cells and normal cells has led to p53 becoming one of the most important ansd rational targets for cancer therapy.

Over the past couple of decades, studies have revealed p53 structure, function, and role in tumorigenesis and development [1-3]. These fundamental studies strongly support the design and development of new approaches for targeting p53 (mutant and wild-type) in cancer therapy. Delivery of wild-type p53 by adenovirus infection kills cancer cells and suppresses tumor growth in pre-clinical and clinical studies [4]. Peptides targeting p53 can rescue p53 function and reduce tumor proliferation [5]. Small molecules targeting 
mutant p53 are under clinical evaluation in different clinical trials [6]. Immunotherapy targeting mutant p53 to suppress tumor growth is becoming a promising approach in cancer therapy. These pioneering studies shed light on prospective cancer therapy that targets mutant and wild-type p53.

\section{2. p53 structure and mutations exploited for drug development}

The frequency of p53 mutations is approximately $50 \%$ across all cancers, however, this frequency varies greatly depending on the cancer type. p53 mutations are most common in ovarian (47.8\%), colorectal (43.2\%), esophageal (43.1\%), head \& neck (40.6\%), and laryngeal (40.4\%) tumors, while cervix (5.8\%), hematopoietic (12.7\%), and endocrine gland (14.6\%) tumors experience the lowest frequency of p53 mutations [7]. High-grade serous ovarian cancer has a p53 mutation rate approaching 95\%.

p53 mutations generally occur in the DNA-binding domain with about $30 \%$ arising in specific hotspots including R175, G245, R248, R249, R273, and R282 (individual mutation rates range from $\sim 3-7 \%$ ) [8]. Clinical studies demonstrate that in many tumor types, the presence of mutated p53 correlates with a worse patient prognosis as compared to the presence of wild-type p53. Though this is still a topic of controversy in some contexts, it is most widely accepted that tumors with mutated p53 predict worse clinical outcomes in breast, head and neck, liver, hematopoietic, and lymphoid system cancers as compared to their wild-type counterparts [9]. One factor contributing to the complexity of this topic is that p53 mutation or variant sequences can have unique effects on cellular function. For example, cancer-associated polymorphisms in codon 72 of TP53 can result in a non-conservative amino acid change to either proline (p53pro) or arginine (p53arg), which have different characteristics. p53pro is less efficient in inducing apoptosis compared to p53arg, while p53arg is more likely to be degraded by the human 
papillomavirus (HPV) E6 protein. Much work by Maureen Murphy has uncovered racial, ethnic, and geographic differences in the codon 72 polymorphism and others. Other cancer-associated mutations in TP53 are most often missense mutations in the central DNA-binding domain. These mutations also vary greatly in terms of their impact on p53 function. Some mutations cause loss of p53 function, some provide p53 the ability to inactivate wild-type p53 that is expressed from the remaining wild-type allele (dominantnegative p53 mutations), and still others cause p53 to acquire oncogenic GOF properties [10]. These unique characteristics of different p53 mutants make mutant p53 a challenging drug target and also necessitate consideration of individual mutations when using mutant p53 as a prognostic biomarker [9].

\section{1 p53 structure exploited for drugs reactivating p53 signaling}

Wild-type p53 consists of a p53 transactivation domain at its $\mathrm{N}$-terminal region and a tetramerization domain at its $\mathrm{C}$-terminal region. The $\mathrm{N}$ - and $\mathrm{C}$-terminals flank the DNA binding domain, also called the core domain [3].

\section{DNA-binding domain}

Amino acid residues $94-292$ of the p53 protein contain the DNA-binding domain that is responsible for p53 binding in a DNA sequence-specific manner to gene regulatory regions to control gene expression. Functionally significant upregulated target genes include p21 (WAF1) which regulates the cell cycle, Bax, DR5, Puma and Noxa which regulate cellular apoptosis in response to cellular stresses, TIGAR which regulates processes related to cellular metabolism, and IRF5/9 which regulates processes related to intracellular immune functioning [1].

\section{P53 DNA-binding response element}


p53 binds specifically to the consensus DNA sequence (5'-RRRCWWGYYY-(N=0-13)RRRCWWGYYY-3') [11]. This discovery has been widely applied to engineer different vectors expressing reporter genes under the control of p53 response elements, in addition to predict endogenous p53 DNA-binding response elements. Use of p53 transcriptional activity reporters has provided a useful tool in the high-throughput screening (HTS) of p53-targeting drugs via functional cell-based assays. Our lab has generated this system and has used this tool to identify a series of small molecules that reactivate the downstream p53 pathway in tumors with mutated p53 [12]. Multiple small molecular weight chemical compounds targeting p53 (wild-type and mutant) have been identified using this method such as NSC59984 [13], prodigiosin [14], and CB002 [15, 16].

\section{Tetramerization domain}

The p53 tetramerization domain is located at the C-terminus between residues 325-355 in the human p53 protein. p53 binding to specific DNA sequences is regulated by tetramer formation via this tetramerization domain. The C-terminus is also one of the major immunodominant epitopes of human p53 that can be recognized by anti-p53 antibodies in the sera of cancer patients $[17,18]$.

\section{$\underline{\text { Transactivation domains of } \mathrm{p} 53}$}

The p53 transactivation domains are located near the $\mathrm{N}$-terminus of $\mathrm{p} 53$. This domain recognizes and binds to the transcriptional coactivators P300/CBP and components of transcriptional machinery. This more $\mathrm{N}$-terminal transactivation domain of $\mathrm{p} 53$ binds to MDM2, an E3 ligase [19]. MDM2 ubiquitinates p53 and this results in p53 degradation via the proteasome. Regulation of p53 by MDM2 keeps basal p53 levels low or undetectable in cells under physiological conditions. The transcriptional domain region of p53 is considered an important domain to target in the development of effective drugs that 
reactivate p53, which led to the discovery of MDM2 inhibitors such as those in the Nutlin family. Small molecule MDM2 inhibitors that activate p53 do so without causing DNA damage as occurs with cytotoxic DNA damaging chemotherapy or radiation. This domain is modulated by post-translational modifications after DNA damage such as phosphorylation and acetylation via cross-talk with many signaling pathways. This provides new insight in the design of treatments targeting p53 in combination with other signals to improve efficacy of cancer therapy. There is a second transactivation domain in p53 that is more $\mathrm{C}$-terminal to the $\mathrm{N}$-terminal domain and includes serine 46 which has been implicated in activation of apoptosis.

\section{C-terminal regulatory domain}

The C-terminal regulatory domain is located at residues 356-393, just following the tetramerization domain. Post-translational modifications are also found in this domain, such as phosphorylation, acetylation, methylation, and ubiquitination. Small molecular compound Toverns is an example of a drug which induces p53 acetylation in this region [20].

\subsection{Conformational changes of p53 mutants utilized for drugs that restore wild-type}

\section{function}

Most missense mutations in p53 occur in the core domain. The wild-type p53 core domain is unstable with low thermodynamic and kinetic stability which allows rapid cycling between folded and unfolded states. Mutations in such residues such as Arg273 and Arg282 destabilize the core DNA-binding domain by enhancing thermodynamic and kinetic instability of mutant p53. These effects are often exploited to design ligands that selectively bind to the native state of the p53 protein to reverse the thermodynamic and kinetic denaturation consequences of these mutations. 
Mutation at Arg249 of p53 results in impaired DNA-binding due to enhanced flexibility of the L3 loop. Mutation at Y220 causes cavity formation in the DNA binding core which causes loss of p53-DNA binding affinity. These mutations have been exploited in drug development. PhiKan083 is a representative example. It was identified to bind to mutant p53 (Y220C) which raises the melting temperature of the mutant and slows down its rate of denaturation [21]. SCH529074 is another example of a small molecule designed based on the DNA-binding domain [22].

Different from other mutations in the core domain, $\mathrm{R} 175 \mathrm{H}$ has been described as a zincbinding mutant. Zinc ions are important in coordinating with C176, H179, C238 and C242 in the core domain to ensure proper folding of the p53 protein [23]. The mutation $\mathrm{R} 175 \mathrm{H}$ next to $\mathrm{C} 176$ reduces zinc ion binding to the coordinate sites, resulting in mutant p53 protein misfolding which abolishes DNA binding. Small molecule NSC319726 (ZMC1) plays a role as a zinc metallochaperones in the stabilization of mutant p53-Zn2+ interaction, resulting in a wild-type conformational change [24].

Mutations at the C-terminus disturb p53 tetramer formation. For example, mutations at Arg337 and Asp352 stabilize the tetramer, and mutation at Arg337 (R337H) disrupts the inter monomer salt bridge, resulting in impaired tetramer formation [25]. However, it is known that not all p53 mutants are equal. The wide variety of mutations in p53 make it challenging to design drugs that target mutant p53 because different mutations might lead to specific structural changes which may need unique small molecules to fit in.

Promising pharmaceutical strategies using small molecular compounds such as Nutlins, RITA, PRIMA-1, and SJ172550 are being tested as monotherapy or as part of a combinations in specific tumor types [26]. 


\section{3 p53 transgenic mouse models (p53 knockout, mutant p53 knock-ins and inducible models) provide a powerful tool to investigate p53 function}

Cell culture in vitro models mostly used for cancer research provide insights into fundamental biological functions of p53, but do not faithfully reproduce events of tumorigeneses inside living tissues. Mouse p53 is highly homologous to human p53, therefore, p53 transgenic mouse models have been widely applied to explore wild-type and mutant p53 biological functions at physiological conditions and address the mechanism of p53 (wild-type or mutant) action on tumorigenesis and drug resistance in vivo. A broad range of mutant p53 knock-ins or p53 knockout mouse models have been generated. In addition to the constitutive germline-knockin or knockout of p53, a Cre-LoxP system (Cre acts as a site specific recombinase to catalyze recombination between specific LoxP sites, and thus depletes genes or gene fragments flanked by LoxP sites) has been applied to conditionally knock in or knock out p53 in mice and to address the role of p53 at different tumor developmental stages [27]. Tissue-specific transcription factor promoters have been exploited for generating tissue-specific expression of Cre which leads to mutant p53 expression or knockdown of p53 only in the specific tissues [28], therefore, providing an opportunity to investigate the role of mutant p53 or p53 loss in sporadic tumor tissues during tumorigenesis and tumor development. These p53 knockins and knockout mouse models mimic initiating events in tumorigenesis and progression of human tumors such as tumor behaviors (tumor growth, metastasis, metabolism, and senescence) and the host immune response in the tumor microenvironment due to $\mathrm{p} 53$ loss or p53 mutations. 
1) Studies of p53 using p53-knockout mouse models demonstrate that p53 is a tumor suppressor. The early studies of p53 using p53 germline-knockout mice with different genetic background such as 129/sv, and C57BL/C showed that P53-/- mice developed tumors earlier than p53+/-mice. T-cell lymphoma was mostly found in p53-/- mice, while osteosarcomas, soft tissue sarcomas, and lymphoma were the tumors that mostly developed in P53+/- mice [29]. p53 germline-knockout renders mice susceptible to carcinogen-induced tumors [30]. Studies of p53 function using p53 knockout mouse models have demonstrated that p53 loss of function (LOF) increases the incidence of carcinogen-induced tumorigenesis of different tumors [31-36]. For example, p53 (-/-) knockout mice are more susceptible than p53(+/-) or p53 (+/+) mice to $\mathrm{N}$-methyl-Nnitrosourea stomach carcinogenesis [37]. Conditional knockout of p53 in murine enterocytes is insufficient to initiate intestinal tumorigenesis but markedly enhances carcinogen-induced tumor incidence and leads to invasive cancer and lymph node metastasis [31]. It is well known that p53 is a stress sensor and functions as a guardian of the genome by regulating cell cycle arrest and DNA repair or inducing cell death if there is too much unrepaired damage. p53 deficiency leads to genome instability, and facilitates causation of malignant tumorigenesis in response to cellular stresses such as hyperproliferation, DNA damage, hypoxia, and inflammation signals. Recently, p53-null mouse models showed that the tumor microenvironment can be shaped by p53. p53 loss changes the senescence-associated secretory phenotype (SASP), leading to suppression of the immune response by recruitment of immune inhibitory cells, which promotes tumor growth. For example, pharmacological activation of p53 in vivo unleashed the interferon program, promoted T-cell infiltration and significantly enhanced the efficacy of checkpoint therapy in a xenograft tumor model [38]. Consistently, p53-null mice showed an accumulation of suppressive regulatory $T$ (Treg) cells in the tumor 
microenvironment [39]. These fundamental discoveries based on P53-knockout mouse models demonstrate that $p 53$ is a tumor suppressor via different mechanisms of p53 action. Conditional regulation of p53 expression in mouse tissues showed that the restoration of p53 expression suppressed lymphomas and sarcomas growth via cellular apoptosis in different mouse models [40,41]. These studies provide a rationale for restoration of p53 in cancer therapy, and multiple pharmaceutical approaches to reactivate p53 are under clinical evaluation for targeting therapy and will be discussed in this review.

\section{2) Mutant p53 knock-in mouse models demonstrate mutant p53 gain-of-function}

(GOF). p53 missense mutations in the germline were found in patients with Li-Fraumeni syndrome and were associated with cancer development. Patients with Li-Fraumeni syndrome with mutated p53 show tumor onset much earlier than patients with p53 deficiency. p53R248Q/+ patients with Li-Fraumeni syndrome have higher tumor numbers and shorter tumor-free survival by 10.5 years than p53 null/+ patients [42]. This clinical observation was also found in the mice with knock-in of mutant p53R172H (mimic of human p53R175) and mouse p53 R270H (mimic of human mutant p53 R273). These mutant p53 knock-in mice showed greater tumor development and metastases as compared to the p53 null mice in the c57BL/6 and 129/Sv backgrounds, indicating a Gainof-function of mutant p53 [43, 44]. p53 mutant knock-in mouse models further support the hypothesis of the unequal functions of p53 mutations. Mutant p53 Knock-in mice harboring the hot spot alleles R248Q and G245S showed their different impacts on tumor development. R248Q/- mice had accelerated onset of all tumor types and shorter survival compared with p53-null mice, but G245S/- mice were similar to null mice in tumor latency and survival, suggesting that mutant p53 GOF alleles are not biologically equal [42]. Conditional knockdown of mutant p53 in cancer cells showed extended mouse survival 
and the tumors underwent apoptosis and tumor regression [45]. These studies suggest that tumors are addicted to the sustained high levels of mutant p53. These fundamental discoveries of mutant p53 GOF provides a rationale for depleting mutant p53 in tumors as a strategy for cancer therapy. Targeting mutant p53 degradation by HSP90 inhibitors significantly extended the survival of mutant p53 (R248Q allele2, and R172H allele3) mice as compared to p53-/- littermates [45]. Drug development targeting mutant p53 degradation is a promising strategy for cancer therapy.

\section{Strategies for boosting wild-type p53 activity in cancer: gene therapy, cytotoxic chemotherapy, MDM2/MDMX inhibitors, p53-binding compounds, targeting p53 PTMs}

Chemotherapies used for the treatment of cancer include DNA damaging agents such as doxorubicin, 5-FU, irinotecan, actinomycin D, etoposide, mitomycin D, bleomycin, daunomycin, and cisplatin. These agents induce the DNA damage response and p53, ultimately resulting in apoptosis mediated by p53 target genes. A majority of clinical studies have demonstrated a correlation between adverse clinical outcomes after treatment with chemotherapy in patients with mutant p53-expressing tumors compared to patients with wild-type p53 tumors. Thus, it is thought that wild-type p53 function is at least partly responsible for clinical efficacy of conventional chemotherapy [46]. Treatments such as conventional chemotherapy and $\mathrm{y}$-radiation activate p53, which mediates apoptosis through activation of a subset of p53 target genes such as Puma, Noxa, Bax, and death receptor 5 (DR5), among others [47]. Though effective, these conventional chemotherapies induce DNA damage that in some cases leads to secondary malignancies, therefore novel strategies for specifically targeting p53 are needed [48]. Despite this, there are no FDA-approved therapies that target either wild- 
type or mutant $\mathrm{p} 53$, though some are in various stages of clinical trials $[26,49,50]$. Advanced strategies including biotherapeutic and pharmaceutic approaches have been developed for targeting p53 reactivation for cancer therapy (Figure 1). Biotherapeutic approaches mostly focus on replacement of p53 with wild-type p53 by gene delivery. Wild-type p53 can be transferred into cancer cells to replace endogenous p53 function using a recombinant virus such as a recombinant adenovirus which fails to replicate efficiently with the E1B-55 Kd protein deletion in cells. Gene therapy based on p53 delivery is under clinical evaluation (Table 1). Pharmaceutical approaches using small molecules for reactivation of wild-type p53 function is a major effort for cancer therapy targeting wild-type p53.

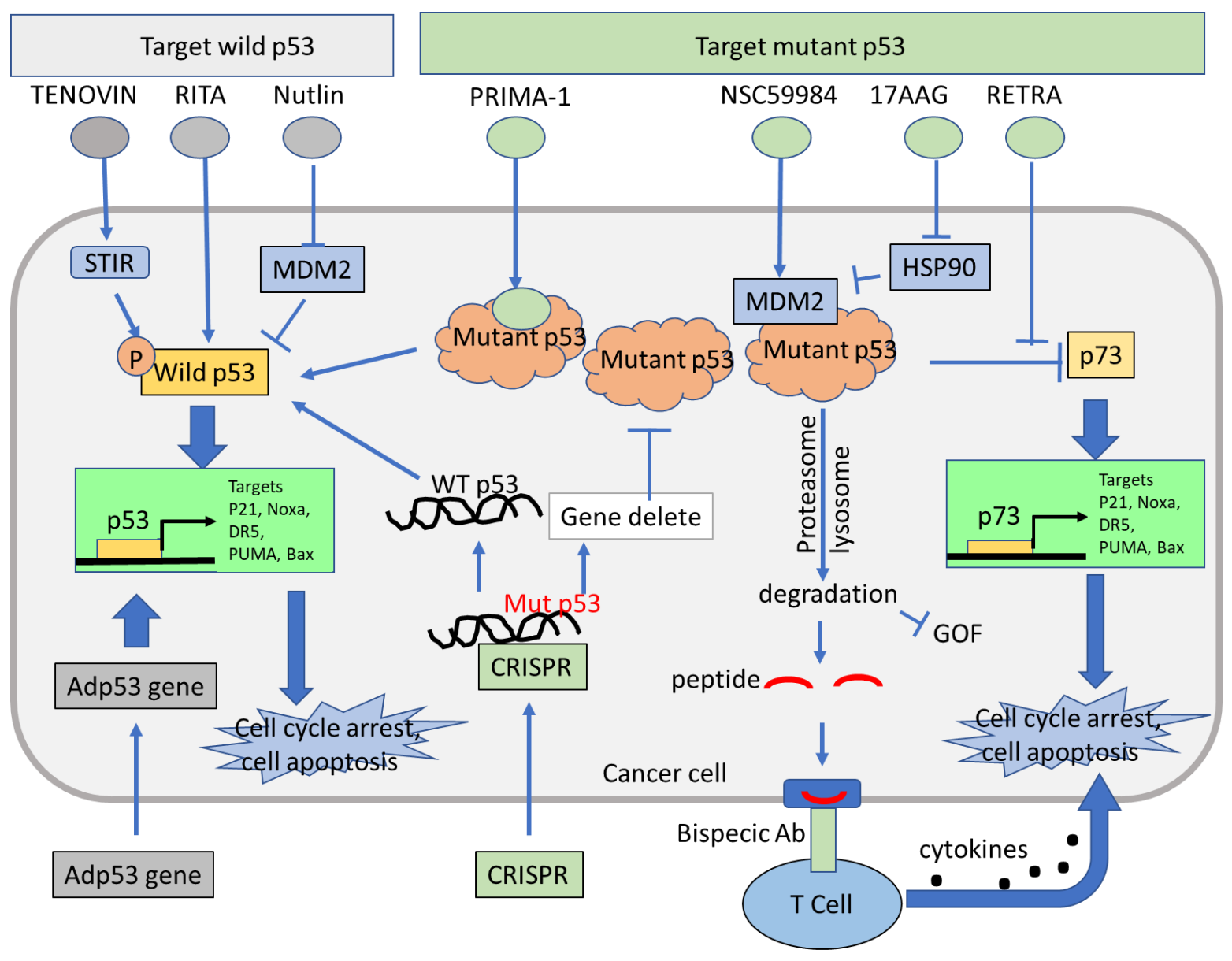


Figure 1. Strategies for targeting mutant p53 and wild-type p53 in cancer cells. Pharmacological approaches for targeting wild-type and mutant p53 in cancer cells are focused on small molecules (upper panel). Small molecules targeting wild-type p53 activation via binding to p53 (such as RITA), inhibition of MDM2 (such as Nutlins), posttranslational modifications (such as tenovin). Small molecules target mutant p53 via restoration of p53 function (such as PRIMA-1), degradation of mutant p53 via activation of MDM2, (such as 17AAG and NSC59984) or interruption of mutant p53-p73 interaction (such as RETRA). Activation of p73 upregulates p53 target gene expression and induces cell death. Biotherapeutic approaches are based on gene transfection and genomic modifications (bottom panel). p53 is transfected into cancer cells with an adenovirus to replace mutant p53, and upregulates p53 signaling (such as rADp53). Genomic editing is used to restore wild-type p53 or delete mutant p53 in cancer cells by genome editing approaches (such as CRISPR). Bispecific antibody with mutant p53-specific peptide and ALH ligands promotes $\mathrm{T}$ cells to recognize and kill p53-mutant tumor cells in cancer immunotherapy.

Table 1. Overview of mutant-p53 targeting and wild-type p53 activating agents.

Abbreviations: HSP40, heat shock protein 40; MDS, myelodysplastic syndrome; MQ, methylene quinuclidinone; p73, tumor suppressor protein p73 (p53 family member).

Data reviewed from references [50-55].

\begin{tabular}{|c|c|c|c|c|c|c|}
\hline \multicolumn{3}{|c|}{$\begin{array}{l}\text { Compound /peptide / } \\
\text { antibody }\end{array}$} & $\begin{array}{l}\text { Chemical name } \\
\text { and/or class }\end{array}$ & Target/Mechanism & $\begin{array}{l}\text { Clinical } \\
\text { development }\end{array}$ & $\begin{array}{l}\text { Refer } \\
\text { ences }\end{array}$ \\
\hline 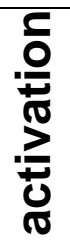 & 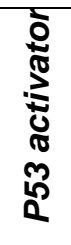 & RITA & & $\begin{array}{l}\text { Binds to p53 and prevents } \\
\text { WT p53 degradation by } \\
\text { blocking interaction with } \\
\text { MDM2 }\end{array}$ & $\begin{array}{l}\text { Experimental } \\
\text { and/or } \\
\text { preclinical }\end{array}$ & {$[56]$} \\
\hline 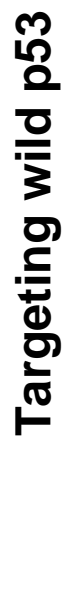 & 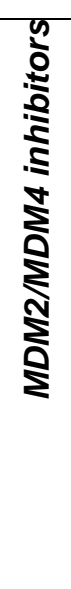 & $\begin{array}{l}\text { Nutlin-3a } \\
\text { RG7112 } \\
\text { RG7388 } \\
\text { RG7775 } \\
\text { MI-77301 } \\
\text { AMG232 } \\
\text { SAR405838 } \\
\text { MK-8242 } \\
\text { CGM097 } \\
\text { DS-3032b } \\
\text { ALRN- 6924 } \\
\text { HDM201 }\end{array}$ & $\begin{array}{l}\text { Cis-imidazoline } \\
\text { Cis-imidazoline } \\
\text { Cis-imidazoline } \\
\text { Pegylated prodrug } \\
\text { idasanutlin } \\
\text { Spirooxindole } \\
\text { Piperidinone } \\
\text { Piperidinone } \\
2(1 \mathrm{H})- \\
\text { Pyrimidinone } \\
\text { Dihydroisoquinolin } \\
\text { one } \\
\text { Unknown }\end{array}$ & $\begin{array}{l}\text { Blocks the interactive } \\
\text { binding sites of p53 and } \\
\text { MDM2, dramatically } \\
\text { increasing the half-life of } \\
\text { p53 and activating p53- } \\
\text { mediated transcription. }\end{array}$ & $\begin{array}{l}\text { The listed } \\
\text { inhibitors } \\
\text { except nutlin-3a } \\
\text { have } \\
\text { undergone or } \\
\text { are currently } \\
\text { undergoing } \\
\text { clinical trials }\end{array}$ & $\begin{array}{l}{[51,} \\
57]\end{array}$ \\
\hline
\end{tabular}




\begin{tabular}{|c|c|c|c|c|c|c|}
\hline & & & $\begin{array}{l}\text { Stapled peptide } \\
\text { Imidazopyrrolidino } \\
\text { ne }\end{array}$ & & & \\
\hline & $\begin{array}{l}\text { on } \\
\frac{0}{0} \\
\frac{\pi}{0} \\
\frac{\pi}{0} \\
\frac{0}{d} \\
\frac{d}{0} \\
\text { N } \\
\sum \\
\text { L }\end{array}$ & $\begin{array}{l}\text { PROTAC 8, } \\
\text { A1874* }^{*}\end{array}$ & $\begin{array}{l}\text { IMiD-based } \\
\text { MDM2 }\end{array}$ & $\begin{array}{l}\text { Targeted degradation of } \\
\text { MDM2 using proteolysis } \\
\text { targeting chimeras } \\
\text { (PROTACs) }\end{array}$ & $\begin{array}{l}\text { Experimental } \\
\text { and/or } \\
\text { preclinical }\end{array}$ & $\begin{array}{l}51, \\
58]\end{array}$ \\
\hline & 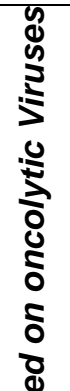 & ONYX-015 & $\begin{array}{l}\text { Recombinant } \\
\text { adenovirus with } \\
\text { wild-type p53 (Ad- } \\
\text { p53) }\end{array}$ & $\begin{array}{l}\text { A mutant adenovirus with } \\
\text { a deleted E1B-55Kd gene } \\
\text { commonly fails to replicate } \\
\text { efficiently in cells with a } \\
\text { wild-type p53 but } \\
\text { replicates in many (but not } \\
\text { all) cells with a mutant p53 } \\
\text { gene. }\end{array}$ & In clinical trials & $\begin{array}{l}{[51} \\
59 \\
60]\end{array}$ \\
\hline & 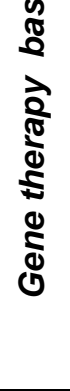 & $\begin{array}{l}\text { Gendicine } \\
\text { (Ad-53) }\end{array}$ & $\begin{array}{l}\text { Recombinant } \\
\text { adenovirus } \\
\text { engineered to } \\
\text { express } \\
\text { wildtype-p53 } \\
\text { (rAd-p53) }\end{array}$ & $\begin{array}{l}\text { Gene replacement } \\
\text { (gene therapy) }\end{array}$ & $\begin{array}{l}\text { Approved in } \\
2003 \text { by the } \\
\text { China Food } \\
\text { and Drug } \\
\text { Administration } \\
\text { (CFDA) to } \\
\text { treat head and } \\
\text { neck cancer }\end{array}$ & $\begin{array}{l}{[51} \\
61 \\
62]\end{array}$ \\
\hline \multirow{4}{*}{ 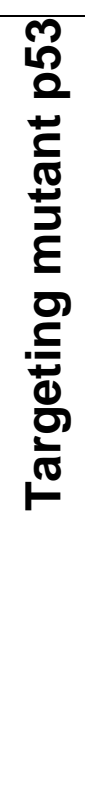 } & \multirow{4}{*}{ 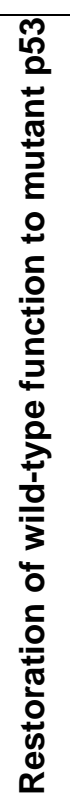 } & CP-31398 & Styrylquinazoline & $\begin{array}{l}\text { Cystein-binding } \\
\text { compounds, Michael } \\
\text { acceptor binding to mutant } \\
\text { p53 }\end{array}$ & $\begin{array}{l}\text { Experimental } \\
\text { and/or } \\
\text { preclinical }\end{array}$ & [63] \\
\hline & & PRIMA-1 & Quinuclidinone & $\begin{array}{l}\text { Cystein-binding } \\
\text { compound is converted } \\
\text { to } M Q \text {, which binds } \\
\text { mutant p53 by Michael } \\
\text { addition }\end{array}$ & $\begin{array}{l}\text { Experimental } \\
\text { and/or } \\
\text { preclinical }\end{array}$ & $\begin{array}{l}{[64,} \\
65]\end{array}$ \\
\hline & & APR-246 & Quinuclidinone & $\begin{array}{l}\text { Cystein-binding compound } \\
\text { is converted to MQ, which } \\
\text { binds mutant p53 by } \\
\text { Michael addition }\end{array}$ & $\begin{array}{l}\text { Phase Ib/ll for } \\
\text { ovarian cancer, } \\
\text { MDS and } \\
\text { oesophageal } \\
\text { cancer }\end{array}$ & [66] \\
\hline & & MIRA-1 & Maleimide & $\begin{array}{l}\text { Michael acceptor } \\
\text { binding to mutant p53 }\end{array}$ & $\begin{array}{l}\text { Experimental } \\
\text { and/or } \\
\text { preclinical }\end{array}$ & [67] \\
\hline
\end{tabular}




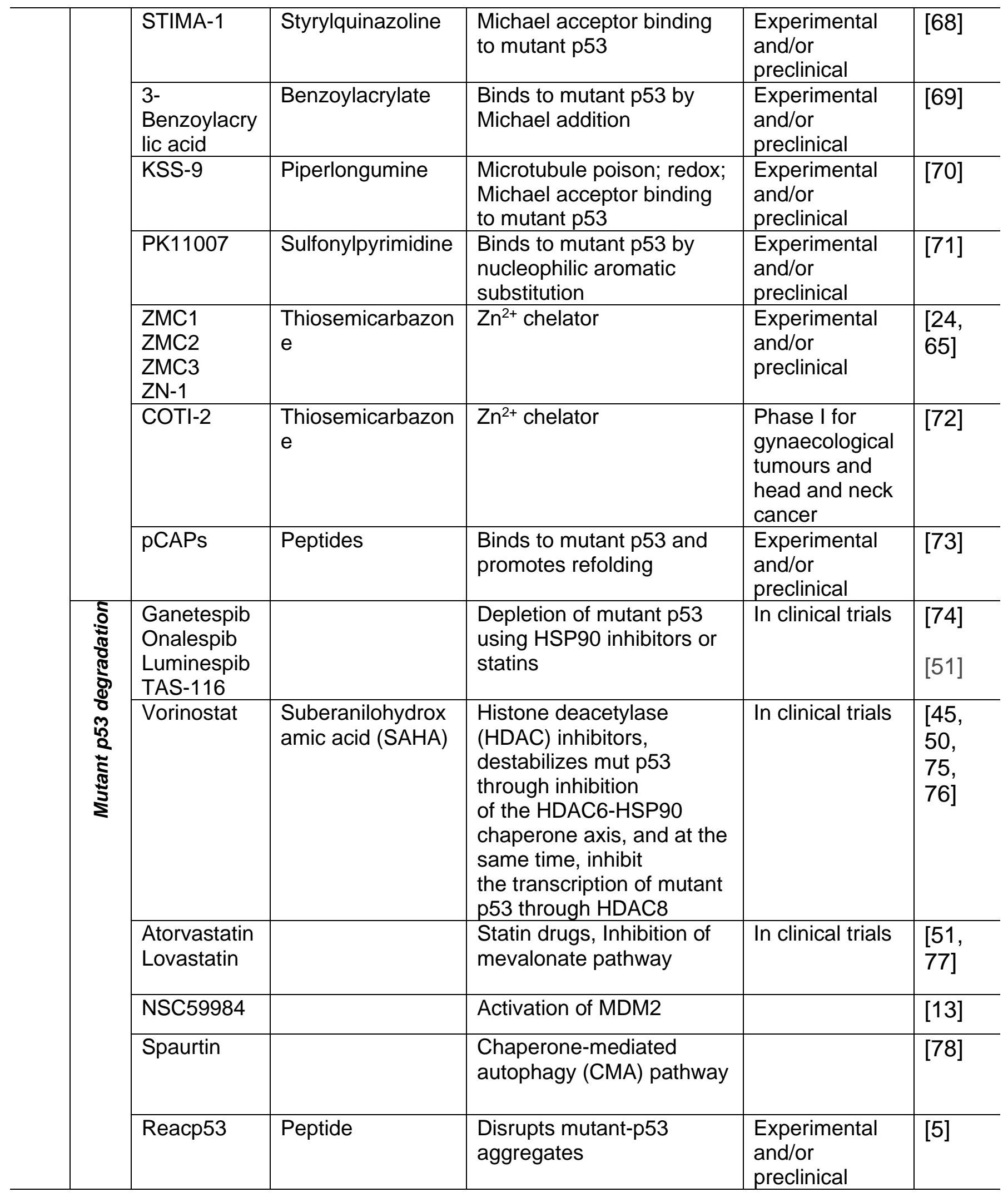




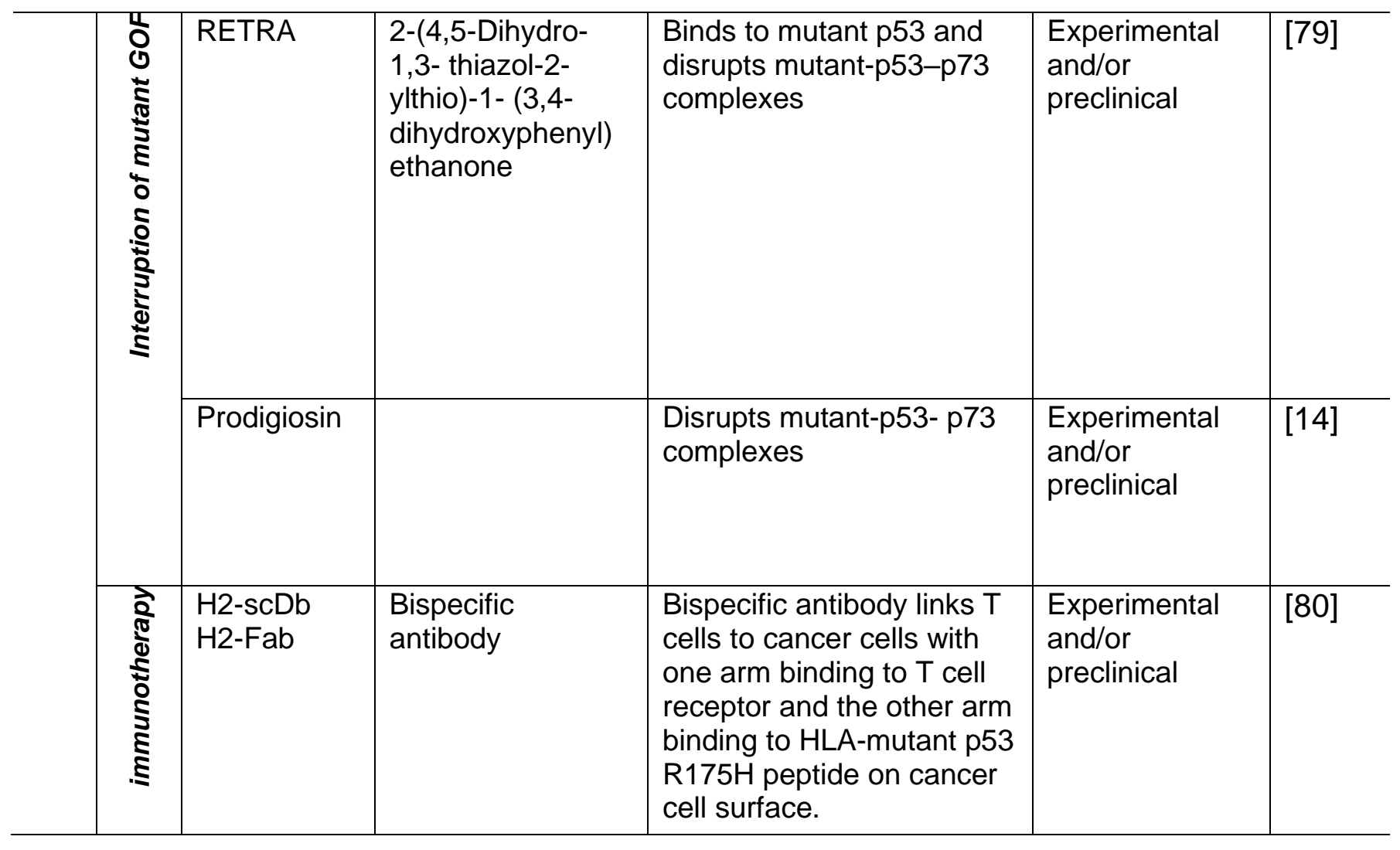

\subsection{Small molecules can directly target wild-type p53 protein}

p53 is a DNA-binding transcription factor that has been characterized as an "undruggable" target, therefore, searching for drugs directly targeting p53 is challenging. Moreover, because the mutations in p53 cause loss-of-function, directly targeting the protein for functional restoration is challenging. Small molecular compound RITA provides a possibility to directly target p53. RITA was first discovered via cell-based screening in human colon carcinoma lines HCT116 and HCT116 TP53-/- [56]. It directly binds to p53 and interrupts p53 interaction with MDM2, therefore preventing p53 degradation. Further studies showed that RITA treatment leads to a p53-dependent global transcriptional response. RITA suppresses oncogene expression and increases tumor suppressor expression via p53 reactivation, leading to p53-mediated cell death [81]. However, RITA treatment also causes genotoxicity through the integrated stress response (ISR) in wildtype p53 cells as well as accumulated ROS in cancer cells [82-84]. ROS-dependent 
activation of c-Jun N-terminal kinase (JNK) plays a crucial role in RITA treatment-induced p53-mediated inhibition of oncogenes leading to cellular apoptosis [84]. Recent studies show that RITA treatment suppresses tumor growth in p53 defective cancer cells [84-86], suggesting non-specificity of RITA in reactivation of $\mathrm{p} 53$.

\subsection{Small molecules can activate p53 via targeting MDM2}

Inhibition of MDM2 is one of the most promising strategies for targeting wild-type p53 activation. Structure-based screening has led to the identification of a series of small molecules which specifically bind to the p53 protein-binding site in MDM2. Several MDM2 or MDM2/MDMX inhibitors have been developed recently and most of them are under clinical evaluation in different clinical trials (Table 1). Nutlins are a representative example of a class of MDM2 inhibitors. Nutlins are cis-imidazoline analogs and were discovered by biochemical screening methods by the pharmaceutical company Roche in Nutley, New Jersey [87]. Nutlin-3 binds to MDM2 and disassociates MDM2 from p53, thereby rescuing p53 from degradation. In preclinical treatment, induction of apoptosis by nutlin-3 seems to be specific to wild-type p53. Recently, additional compounds in the Nutlin family have been developed which also inhibit MDM2, such as RG7112 [88] and RG7338 [89]. MDM2 inhibitors show specific and potent antitumor effects in various tumors carrying wild-type p53 in preclinical and clinical studies. For example, RG7112 demonstrated clinical activity against relapsed/refractory acute myeloid leukemia (AML) and chronic myelogenous leukemia (CLL). MDM2 inhibition stabilizes wild-type p53 and transcriptionally upregulates gene expression of p53 targets in different tumors such as AML [90-92]. In addition to Nutlins, AMG232, a selective piperidinone inhibitor of the MDM2-p53 proteinprotein interaction also causes upregulation of p53 signaling and has a potent antitumor 
effect [93]. Another MDM2 inhibitor DS-3032b (Rain-32/Milademetan) has entered clinical trials for MDM2-amplified cancers including Merkle cell cancers among others.

Inhibition of MDM2 and MDMX is considered a potent antitumor approach via activation of p53. MDMX as a paralogue of MDM2 and is involved in p53 degradation by augmenting MDM2 activity [94]. Combination treatment with MDM2 and MDMX inhibitors has been investigated since the first MDMX inhibitor SJ172550 was discovered. SJ172550 binds the p53-binding pocket of MDMX and effectively kills retinoblastoma cells which have high expression of MDMX [95]. Treatment with SJ-172550 combined with MDM2 inhibitors additively suppresses tumor growth [95]. Similar results were also observed after combination treatment with nutlins and XI-011 (NSC146109), an MDMX inhibitor $[80,87]$, suggesting that dual inhibition of MDM2/MDMX causes a more potent antitumor effect and activation of p53 compared to treatment with either alone. Compounds that dually inhibit MDM2 and MDMX have been developed, such as ALRN6924 [96]. ALRN-6924 is cell-penetrating stapled a-helical peptide which robustly activates p53-dependent transcription by preventing its interaction with MDM2/MDMX and induces cell cycle arrest and apoptosis in TP53 wild-type tumors [96]. Clinical evaluations at phase I show that ALRN-6924 is well tolerated and demonstrate anti-tumor activity [97].

\subsection{Small molecules can induce p53 transcription via post-translational modifications}

Post-translational modification is one of the key steps for p53 activation as a transcription factor. Small molecule Tenovins was identified as a p53 activator via acetylation of p53. Tenovins blocks deacetylation of SirT1 substrates, including p53, and enhances p53dependent transcription in cells through SirT1 inhibition [20]. Phosphorylation of p53 at 
different sites in the C-terminus affect p53 functions. Small molecule Curaxin phosphorylates p53 at Ser392 through casein kinase 2 [98]. Topln treatment results in phosphorylation of p53 at Ser 15 and stabilizes the p53 protein by dissociation of MDM2 and p53 [99]. These studies provide a rationale for therapeutic targeting of posttranslational modification of p53 to activate its transcriptional function. It is recognized that there is potential for non-specific effects of these small molecular weight compounds, since these kinases are not p53-specific. Even so, upregulation of p53 via posttranslational modifications can be exploited in combination with other therapies which target other parts of the p53 regulatory network to increase efficacy of p53-targeting therapy.

\section{4. p53-dependent and p53-independent strategies for targeting p53 pathway restoration in p53-mutated cancers}

The tumor-specific mutant p53 is an attractive target candidate for cancer therapy development. Small molecules have been used to restore p53 function and inhibit mutant p53 GOF (Figure 1). These pharmacological approaches have been investigated in different tumors carrying mutant p53. In addition, biotherapeutics appear to be potentially powerful approaches for targeting mutant p53 in cancer cells. CRISPR is a representative example for genome modification of mutant p53. Targeting mutant p53 genomic modifications by CRISPR is at its beginning and may soon be able to clinically target mutant p53 for cancer therapy. In this review, however, we focus on pharmacological approaches for targeting mutant p53 in cancer therapy.

\subsection{Restoration of wild-type function in tumors expressing mutant p53}

Restoration of wild-type function in mutant p53-expressing tumors is a promising strategy for cancer therapy. However, restoration of wild-type function of p53 is challenging 
because of the high variation of p53 mutations in cancer. PRIMA-1 (APR-246) is a representative example of a p53-restoring compound. PRIMA-1 (APR-246) is converted to the reactive electrophile methylene quinuclidinone (MQ), which covalently binds to Cys 124 and 277 at the core domain of mutant p53. Binding of MQ causes refolding of mutant p53 and restores wild-type functions $[64,100,101]$. Similar thiol reactivity of mutant p53 was also found with other mutant p53-reactivating compounds, such as MIRA-1, CP-31398, STIMA-1 and 3-benzoylacrylic acid (Table 1). They bind to mutant p53 by Michael addition. Restoration of wild-type p53 by p53-reactivating small molecular weight compounds such as PRIMA-1 (ARP-246) induces p53 target gene expression (such as p21, Puma, and Noxa), triggers cell death, and suppresses tumor growth in vivo. APR-246 is the first p53-restoring compound under clinical evaluation either as monotherapy or in combination treatment. APR-246 in combination with azacytidine is under clinical evaluation at phase I and III in acute myeloid leukemia.

Phikan083 and SCH529074 are two small molecules identified via structure-based assays. Both compounds restore wild-type p53 function to mutant p53, but the mechanism is different. Phikan083 is a carbazole derivative identified based on a docking-based screen for a molecule that fits into this cavity. Phikan083 binds to the cavity that results from the Y220C mutation of p53 and restores wild-type function [21]. By contrast to Phikano83, SCH529074 acts as a chaperone and binds specifically to the p53 DNA-binding domain, restoring DNA-binding activity to mutant p53 [102]. A single amino acid change (N268R) abolishes the binding of SCH529074 [102]. Alkylating drugs 3benzoylacrylic acid and its fluorinated derivative (E)-4-(4-fluorophenyl)-4-oxobut-2-enoic acid were identified to covalently bind to the p53 binding domain containing the mutations Y220C, R175H, G245S, R249S, or R282 by increasing the melting temperature of the core domain [69, 71]. 
NSC319726 (ZMC1) is a small molecule zinc metallochaperone that specifically refolds mutant p53 (R175) to the wild-type conformation [24]. ZMC1 facilitates zinc ion binding to mutant p53 and leads to wild-type-like conformation changes and restores wild-type-like functions. ZMC1 upregulates p53 target expression and induces cellular apoptosis in cancer cells harboring the R175 mutation.

\subsection{Therapeutic induction of mutant p53 degradation}

Mutant p53 is stabilized in most cancer cells. Cancer cells are addicted to mutant p53 for their survival and proliferation. Preclinical studies showed that knockdown of mutant p53 extended mouse survival and repressed tumor growth [45], suggesting that depletion of mutant p53 is a promising therapeutic approach. However, genomic depletion of mutant p53 is not yet available for cancer therapy in the clinic. Most studies are focused on pharmacological approaches to induce mutant p53 degradation via the proteosome or lysosome.

One example of a group of compounds that exploit the proteosome to induce mutant p53 degradation are HSP90 inhibitors such as geldanamycin [103] and 17AAG [104]. HSP90 inhibits MDM2 therefore protecting mutant p53 from MDM2-mediated ubiquitination [105]. Inhibition of HSP90 activity releases MDM2 from HSP90 and allows MDM2 to interact with mutant p53. HDAC inhibitor, SAHA, inhibits HSP90 activity, thus resulting in mutant p53 degradation in cancer [106]. These studies suggest that MDM2 is a key factor in mutant p53 destabilization. The role of MDM2 in mutant p53 degradation was exploited by NSC59984 to induce mutant p53 degradation in human cancer cells. NSC59984 is a small molecule that induces mutant p53 degradation via activation of MDM2 [13]. In addition to MDM2-mediated mutant p53 degradation via the proteasome, HSP40-CHIP appears to be a new pathway involved in mutant p53 degradation. Small molecules 
statins (cholesterol-lowering drugs) were found to induce mutant p53 (R175) degradation via HSP40-CHIP [77]. These studies provide a potential strategy to deplete mutant p53 through a mevalonate pathway-HSP40 (DNAJA1) axis.

Spaurtin is a representative example for exploiting the lysosome to induce mutant p53 degradation through the chaperone-mediated autophagy (CMA) pathway [78]. These small molecules induce mutant p53 degradation via interruption of p53 stabilization. It is unclear whether they directly bind to mutant p53. The identification of CMA as a new degradative mechanism for mutant p53 provides the possibility of activating CMA as a new treatment for cancers with mutant TP53.

Recently, proteolysis-targeting chimeras (PROTACS) have been developed to modulate protein degradation. PROTACs are heterobifunctional molecules consisting of one ligand for binding to a protein of interest and another to an E3 ubiquitin (E3) ligase, connected via a linker [107]. This promising technique will provide a new approach for drug discovery by targeting mutant p53 degradation.

\subsection{Activation of $p 73$ to bypassing mutant p53 in restoration of wild-type p53 function}

Mutant p53 forms an inhibitory complex with p73 to abolish the transactivation and proapoptotic activity of p73. p73 is a member of the p53 family and has a similar structure and function to p53. Active p73 can induce cellular apoptosis via regulation of p53-targets. Our lab conducted an early HTS and found that a significant proportion of the selected candidates relied on p73 for p53-reporter activation in p53 mutant cancer cells, providing a rationale for activating p73 thereby bypassing mutant p53 loss-of-function [12].

Interruption of mutant p53 binding to $p 73$ results in release of $p 73$ from the inhibitory complex. Small molecular compound RETRA is an example of a compound that interrupts mutant p53 GOF. RETRA binds to mutant p53, blocks interaction between mutant p53 
and p73, and results in p73 activation [79]. Similar results were also found in treatment with prodigiosin. Prodigiosin promotes p53 pathway activity via releasing p73 from the inhibitory complex with mutant p53 in p53 mutant-cancer cells [14]. RETRA and Prodigiosin produce tumor-suppressor effects through p73-like reactivation of p53.

NSC59984 also can activate the p73 pathway by degradation of mutant p53 [13]. Different from NSC59984, statins were not found to upregulate p73 signaling though they can induce mutant p53 degradation [77]. These studies suggest that release of p73 from the mutant p53 inhibitory complex is one of the steps for p73 activation. p73 activation may need further regulation through multiple signaling pathways. Targeting p73 activation in combination with depletion of mutant p53 GOF, or small molecular weight compounds with the dual effect could be a promising approach for a potent antitumor effect.

\subsection{Restoration of p53 pathway signaling independent of p53 through non- canonical regulatory pathways}

Global gene expression panels show multiple of genes transcriptionally regulated by p53 in a canonical way [108-112]. In addition, p53 pathway signaling was found to partially overlap with the other signaling pathways. It its known that the canonical p53 targets can be regulated via other factors, via a p53 independent manner [113-116]. For example, p21 is a p53 transcriptional target and transcriptionally upregulated via p53 binding to its promoter. In addition, p21 also is regulated via other factors either at the transcriptional level or post translational modifications independent of p53 [117]. Non-canonical pathways also regulate p53 pathway signaling in addition to the canonical p53 regulation. The proof concept of non-canonical regulation of p53 pathway signaling was raised in our recent investigations of p53 pathway restoration. The transcriptomic and proteomic analysis showed that the compounds PG3-Oc, CB002 and their analogues increased 
ATF3/4 transcriptomic and proteomic targets which overlapped with canonical p53 pathway signaling $[118,119]$. The integrated stress response ATF3 and ATF4 transcription factors play a role in the stimulation of pro-apoptotic p53 targets by p53 pathway restoring compounds in tumor cells expressing mutant p53. We found that PG3Oc partially upregulates the p53-transcriptome (13.7\% of public p53 target-gene dataset; $15.2 \%$ of in-house dataset) and the p53-proteome (18\%, HT29; 16\%, HCT116-p53-/-). ATF4 is a key regulator of PG3-Oc-induced p53 pathway restoration. ATF4 shares a subset of p53 target genes involved in cell cycle arrest and apoptosis such as PUMA, DR5, p21, Noxa and NAG--1 in cells in response to PG3-oc [118]. We detected 197 genes in the Fischer p53 dataset (343 genes) and out of those, 102 were differentially expressed by a CB002 analogue in p53 deficient cancer cells, indicating that nearly $50 \%$ of the established p53 target genes were altered [119]. Our investigation suggests that ATF4 is a non-canonical factor to restore p53 pathway signaling independent of p53. ATF3/4 was found to induce NOXA expression by binding to NOXA promoter in head and neck squamous cell carcinoma (HNSCC) in response to cisplatin treatment [114]. These results suggest that p53 pathway signaling can be regulated via non-canonical regulatory pathways (Figure 2). These non-canonical regulation pathways compensate for p53 loss and play an important role to restore p53 pathway signaling in tumor cells that express mutant p53. The p53 canonical targets Noxa and Puma are regulated by ATF3/4 via noncanonical regulatory pathways and are required to induce cell death by CB002 and PG3Oc respectively $[118,119]$. These results provide a therapeutic strategy for modulating components of non-canonical regulatory pathways to achieve partial restoration of the global p53 transcriptome and proteome that include critical p53-effectors of cell cycle arrest, apoptosis, and metastasis in p53-deficent cancer cells. 


Canonical
cellular stress stimuli
Cell cycle arrest $\quad$ Cell death
p21

\section{Targeting p53 function in immunotherapy: bispecific antibodies, gene therapy, small molecule combinations}

Immunotherapy is a revolutionary development for cancer therapy [120]. Mutant p53 and wild-type p53 both relate to the immune response. p53 function related to the immune response provides a possibility for combination treatments with immune checkpoint 
blockade (ICB) in different tumors. With high protein level and tumor specificity, mutant p53 becomes one of most relevant tumor antigens to target with tumor vaccines and immunotherapy.

\subsection{Activation of wild-type p53 for immunotherapy using immune checkpoint inhibitors}

Activation of p53 promotes T-cell infiltration and sensitizes tumor cells to checkpoint therapy. There are some successful examples of activation of p53 in combination with immune checkpoint blockade (ICB) in different tumors. rADp53 in situ gene therapy in combination with Pembrolizumab has shown a potent antitumor effect and prolonged survival in different tumor models [121]. The P53MVA vaccine activates CD8+ cells [122] and the P53MVA vaccine in combination with Pembrolizumab has appeared feasible, safe, and may offer clinical benefit in patients [123]. Similar to the overexpression of wildtype p53 such as rADp53 and P53MVA, MDM2 inhibitor ALRN-6924 shows that activation of p53 boosts the immune response against tumors by activating interferons in mouse models and human patients [124]. p53-restoring compound PRIMA-1 (APR-246) in combination with Pembrolizumab is under clinical evaluation in phase I/II.

\subsection{Targeting mutant p53 in immunotherapy}

CD8+ and CD4+ T-lymphocytes recognize short peptides (epitopes as tumor antigens) derived from proteins presented on the cell surface in association with class I or II human leukocyte antigen (HLA) molecules. High expression of mutant p53 provides a possibility to generate vaccines to prevent tumor growth. However, mutant p53 is localized inside of cells, not on the cell surface.

Recent studies detected mutant p53 peptides presented on the cell surface with HLA complex. This mutant p53 peptide-HLA complex on the cancer cell surface provided a 
possibility for designing a new approach for immunotherapy by enhancing T-cell recognition of cancer cells based on these neoantigens. For this purpose, Dr. Vogelstein and his colleagues developed a new bispecific antibody $\mathrm{H} 2-\mathrm{scDb}$ with one arm binding to a T-cell receptor and the other recognizing the mutant p53(R175) peptide-HLA complex on the cancer cell surface [80]. This bispecific antibody function acts as a bridge between cancer cells and T-cells and stimulates T-cells to kill tumor cells. Treatment with this bispecific antibody specifically targets mutant p53-expressing tumor cells and significantly suppresses tumor growth. The bispecific antibody generated with different p53 mutations will bring T cell-based immunotherapy one step forward toward development of precision cancer medicine.

\section{Combination therapies targeting p53 cell cycle checkpoints, MDM2-p53 inhibition or mutant p53}

Multiple oncogenic and signaling pathway abnormalities in individual cancers promote cancer cell resistance to conventional chemotherapy. Combinatorial treatment with two different drugs with individual targets can enhance antitumor efficacy compared to a mono-therapy approach. This approach is widely used in cancer therapy in the clinic [125]. Clinical studies show effect of AdP53 gene therapy combined with chemotherapy or radiotherapy. These studies provide a rationale for targeting p53 reactivation in combination treatments. p53 activation in combination treatment improves antitumor efficacy and minimizes side effects and newly developed resistance in tumors at different progressive stages.

\subsection{Targeting p53 in combination with cell stress signaling}

ROS stresses are common events in cancer cells. Administration of p53-restoring compounds often results in cell stress and increases cellular ROS levels. Cellular stress 
has been exploited in combination treatment with p53-targeting compounds. PRIMA-1 (APR-246) is one example of a p53-reactivating compound which induces cellular stress that can be exploited with combination treatments. PRIMA-1 (APR-246) was found to increase ROS by depleting GSH. SCL7A11 is component of the $\mathrm{xC}(-)$ system in the regulation of GSH and is considered a major biomarker for APR-246 treatment in cancer [126, 127]. PRIMA combined with a $\mathrm{xC}(-)$ system inhibitor, which increases ROS, promotes the antitumor effect of the combination treatment [128].

Signaling pathways related to cellular stresses can be targeted in combination with p53reactivating compounds. JNK is a signaling pathway related to the stress response and has been found to be activated in p53 pathway targeting treatments. p53 restoring compound PRIMA was found to induce p53-dependent apoptosis through this pathway in colon cancer [129]. Treatment with the p53-reactivating compound RITA demonstrated a significant number of differentially expressed genes associated with the stress response including the JNK signaling pathway. Combined treatment with RITA and a JNK activator synergizes in cytotoxic responses in MM cell lines and patient samples [130]. The mechanism by which small molecules induce p53-mediated apoptosis through the JNK signaling pathway provides a rationale for the combination of p53 activating drugs with JNK activators in the treatment of some types of cancer. These studies propose a new approach for targeting mutant p53 in combination with cellular stress signaling.

\subsection{Targeting p53 in combination with inhibition of Raf/MEK/ERK pathways}

Abnormalities in p53 and the Raf/MEK/ERK axis are among the most common during tumorigenesis and tumor development in various cancers. Targeting p53 in combination with inhibition of the Raf/MEK/ERK pathway is effective in certain drug-resistant cancer cells. Simultaneous blockade of MEK and MDM2 signaling by Nutlin-3a triggered 
synergistic, pro-apoptotic responses in AML cell lines [97]. Similar results were also observed with combination treatment of MDM2 antagonist (RG7388) and MEK inhibitors in dedifferentiated liposarcoma [131]. In melanoma cells, treatment with nutlins combined with inhibitors of ERK2 was found to synergistically induce cell apoptosis. Nutlin-3 combined with vemurafenib (inhibitor of mutant Raf) synergistically induced apoptosis and suppressed melanoma cell viability in vitro and tumor growth in vivo, suggesting that reactivation of p53 by nutlins overcomes vemurafenib resistance in melanoma [132]. Sorafenib, a Raf inhibitor, along with nutlin-3 synergistically induced apoptosis in RCC [133].

These results strongly indicate the therapeutic potential of combined MEK blockade and MDM2 inhibition to activate p53 in cancer cells. A p53-based combinatorial approach can reduce doses and side effects of agents in combinations and efficiently suppresses tumor growth.

\subsection{Targeting p53 (wild-type and mutant) in combination with conventional chemotherapy}

Clinical studies demonstrate improved outcomes after treatment with conventional chemotherapy in patients with tumors harboring wild-type p53 compared to mutant p53, suggesting a crucial role of wild-type p53 in the cellular response to chemotherapy. This idea is supported by the observation that lymphoid cells from p53 knockout mice experience complete chemoresistance [134]. Furthermore, others have observed that wild-type p53 is necessary for cytotoxicity of conventional chemotherapy in vitro [135137]. These findings suggest that overexpression or reactivation of wild-type p53 may synergize with chemotherapy treatment to induce apoptosis in cancer cells, and this has indeed been observed in vitro $[138,139]$. 
p53-targeting small molecules have been investigated for an antitumor effect not only as monotreatment, but also in combination with chemotherapy. Conventional chemotherapeutic agents 5-FU, doxorubicin, etoposide, and cisplatin are often used in combination treatment with p53-targeting agents. p53-based chemotherapy significantly reduces the genotoxic burden and induces high anti-tumor efficacy.

Nutlins have been widely used in treating cancer cells with wild-type p53. Effectiveness of nutlin-3 in combination with chemotherapy has been observed in preclinical studies. Nutlins and their analogs (such as MI-43, MI-63 and MI-319) synergize with genotoxic drugs such as 5-FU, doxorubicin, etoposide, and cisplatin in multiple tumors carrying wildtype p53 such as hematological malignancies, lymphoma, neuroblastoma, hepatocellular carcinoma, lung cancer, and pancreatic cancer. Nutlins in combination with chemotherapy show minimal toxic effects in normal cells. Dual inhibition of MDM2/MDMX by ALRN-6924 enhances antitumor efficacy of chemotherapy in TP53 wild-type hormone receptor-positive breast cancer models [140]. RITA in combination with doxorubicin enhanced sensitivity of NALM-6 cells to doxorubicin and promoted doxorubicin-induced apoptosis. Small molecules targeting p53 activation decrease the necessary dose of conventional chemotherapy to reduce genotoxicity but enhances or magnifies the effects of the chemotherapies.

Targeting mutant p53 in combination with conventional chemotherapy is another approach for increasing antitumor efficacy. Mutant P53 is a contributing factor for drug resistance. p53 mutations have been reported to correlate with resistance to platinum chemotherapy in different types of cancer cells. PRIMA-1 (APR-246) is one of the p53restoring compounds that is administered in combination treatment with conventional chemotherapy [141]. Combination treatment with PRIMA-1 (APR-246) overcomes cancer 
cell resistance to chemotherapy. PRIMA-1 was found to synergistically induce cell death in combination with multiple chemo-drugs such as in combination with Adriamycin in NSCLC cell lines carrying different p53 mutations [142] or in combination with doxorubicin or cisplatin in thyroid cancer cells [143]. APR-246 has been reported to overcome chemoresistance to cisplatin and doxorubicin in p53-mutant ovarian cancer cells [144]. Combination treatment based on targeting the mutant P53 pathway prevents development of drug resistance in cancer cells.

The synergistic effect of p53 overexpression or reactivation and chemotherapy provides a promising new direction for development of combination therapies for patients with tumors containing mutant p53.

\subsection{Combination targeting p53 cell cycle checkpoints in p53 mutated cancer cells}

p53 is involved in the G1-S cell cycle checkpoint via regulation of p21, and arrests cells at the G1 phase to allow enough time for DNA repair or otherwise induces cell death in response to DNA damage. Mutant p53-expressing cancer cells have defective G1 cell cycle checkpoints and this causes such cancer cells to be more reliant on alterative mechanisms to maintain cells survival. The ataxia telangiectasia and Rad3-related kinase (ATR)- Checkpoint kinase 1 (Chk1) axis and WEE1 are compensatory pathways independent of p53 involved in intra-S and G2/M checkpoints in response to DNA damage [145]. These cellular vulnerabilities enforced by mutant p53 can be exploited by further targeting of the checkpoints in response to DNA damage in p53-mutated cancer cells. Accumulating studies show that inhibition of ATR/Chk1/WEE1 sensitizes p53-deficient cancer cells to DNA-damaging agents.

Chk1 has a critical role in the DNA damage response (DDR) and cell cycle checkpoints at $S$ phase and at the G2/M transition [146]. Studies based on in vivo and in vitro 
experiments show that Chk1 inhibitors (such as UCN-01, AZD7762) sensitize p53deficient cancer cells to DNA-damaging agents (such as irinotecan and cisplatin) in different tumor types including TNBC, head and neck cancer, and pancreatic cancer [147149]. Inhibition of Chk1 potentiates the cytotoxicity of DNA damaging agents in p53 deficient cells.

WEE1 is a crucial regulator of the G2/M checkpoint of the cell cycle independent of p53 [150]. Abrogation of the G2/M checkpoint by WEE1 inhibition can sensitize p53-deficient cells to DNA-damaging agents. This is supported by results from experiments with WEE inhibitors in combination with chemotherapy and radiation therapy in p53 mutant cancer cells. MK1775 (Adavosertib, AZD1775) is a small molecular weight WEE1 kinase inhibitor. Blockade of WEE1 by the inhibitor MK1775 synergistically induces cell death in p53 mutant colonic cancer when combined with irinotecan [151]. MK-1775 also synergizes with gemcitabine to suppress tumor growth selectively in p53-deficient pancreatic cancer and ovarian cancer [152, 153]. MK1775 prevents WEE1-mediated phosphorylation CDK1.

WEE1 regulates CDK1 activity at the G2/M checkpoint and prevents entry into mitosis. Inhibition of WEE1 causes mitotic entry without completion of DNA repair and replication upon DNA damage, resulting in mitotic catastrophe and apoptosis. The combination results in significant DNA damage due to failure of DNA repair in the cells which lose both the p53-checkpint at G1 and the WEE1-checkpoint at G2, leading to synthetic lethality. Clinical studies at phase II show a promising antitumor efficiency of the combination of MK1775 with chemotherapy such as carboplatin and paclitaxel in p53-mutated ovarian cancer $[154,155]$.

Many ATR, Chk1 and WEE1 inhibitors have been developed and evaluated in different tumors in clinical trials $[156,157]$. Mutant p53 can be a synthetic lethality factor in cancer 
cells to increase antitumor efficacy of ATR/Chk1/WEE1 inhibitors targeting checkpoints in combination when combined with chemotherapy for cancer therapy.

\section{Summary and prospects}

The tumor-specific p53 status and the important role of p53 in regulation of cell function indicates p53 as a promising target for cancer therapy. Although there is no FDAapproved drug targeting p53 (wild-type or mutant), advanced strategies based on p53 structure and function have continued to be developed for targeting p53 in cancer therapy (Figure 1 and 2), and some of them are under clinical evaluation (Table 1). Among the strategies, MDM2 is considered one of the key factors in drug targeting wild-type p53 or mutant p53. Interruption of p53 binding to MDM2 is one of the promising strategies to reactivate $\mathrm{p53}$. This has led to the identification of MDM2 inhibitors. By contrast, inducing MDM2-mediated ubiquitination of mutant p53 is another promising strategy to induce mutant p53 degradation, and this has pointed to HSP90 inhibitors, HDAC inhibitors, and NSC59984 that activate MDM2 and destabilize mutant p53. Further refinement of desirable effects of these small molecules will facilitate the translation of these promising compounds into clinical practice. Non-specific and off target effects are a common weakness of small molecules when they are applied in cancer therapy. To improve the efficacy and reduce the off target effects, targeting p53 in combination treatment has been widely investigated in preclinical and clinical research as a promising strategy for enhancing antitumor efficacy and reducing toxicity. Searching potential biomarkers will be essential for targeting p53 in combination therapy. Next genomic sequencing and proteomic assays provide global gene and gene expression patterns of tumors derived from patients. This information may be useful for rationally designing therapeutic approaches by targeting p53 and/or in combination with other altered signal pathways as 
additional targets in cancers. The patient-derived tumor organoids or patient-derived xenografts mouse models may become invaluable systems to further identify therapeutic approaches and contribute to personalized cancer medicine.

Besides pharmacological approaches currently developed, biotherapeutic approaches targeting p53 (such as gene therapy and genomic editing) appear as promising strategies in cancer therapy. p53 based gene therapy has been considered a promising approach for cancer therapy as a monotherapy or in combination with chemo- and radiotherapy in a range of tumor types in clinical trials in China $[61,158]$. Selective delivery of wild-type p53 can increase the specificity of p53 gene therapy. Recently, cationic liposomes coated with anti-transferrin receptor single chain $\mathrm{Ab}$ fragment $(\mathrm{scL})$ has been used to help deliver wild-type p53 (scL-53). This scL53 nanocomplex can be specifically delivered to tumors based on the tumor transferrin receptor that is not expressed on normal cells [159]. The scL serves as an example and provides a new approach for p53-based gene therapy with high specificity and low toxicity. Genetic editing of mutant p53 appears to be an attractive strategy for p53-targeting therapy. The CRISPR/Cas9 system has been used to repair the TP53 414delC mutation to the wild-type TP53 genotype and inhibit the cell proliferation of PC-3 cells [160]. The CRISPR/Cas9 system could become a powerful technique which will bring genomic editing of p53 closer to cancer therapy $[161,162]$.

Funding: This work was supported in part by the Teymour Alireza $P^{\prime} 98, P^{\prime} 00$ Family Cancer Research Fund established by the Alireza Family.

Acknowledgments: WE-D. is an American Cancer Society Research Professor and is supported by the Mencoff Family University Professorship at Brown University.

Disclosures: W.S.E-D. is the Founder of p53-Therapeutics. W.S.E-D. has disclosed this relationship and potential conflict of interest to his academic institution/employer and is 
fully compliant with $\mathrm{NIH}$ and institutional policy that is managing this potential conflict of interest. The remaining authors declare no conflict of interest. 


\section{References}

1. Kastenhuber, E.R. and S.W. Lowe, Putting p53 in Context. Cell, 2017. 170(6): p. 1062-1078.

2. Gomes, A.S., et al., Structural and Drug Targeting Insights on Mutant p53. Cancers (Basel), 2021. 13(13).

3. Joerger, A.C. and A.R. Fersht, The tumor suppressor p53: from structures to drug discovery. Cold Spring Harb Perspect Biol, 2010. 2(6): p. a000919.

4. Valente, J.F.A., J.A. Queiroz, and F. Sousa, $p 53$ as the Focus of Gene Therapy: Past, Present and Future. Curr Drug Targets, 2018. 19(15): p. 1801-1817.

5. Soragni, A., et al., A Designed Inhibitor of p53 Aggregation Rescues p53 Tumor Suppression in Ovarian Carcinomas. Cancer Cell, 2016. 29(1): p. 90-103.

6. Lane, D.P., C.F. Cheok, and S. Lain, p53-based cancer therapy. Cold Spring Harb Perspect Biol, 2010. 2(9): p. a001222.

7. Olivier, M., M. Hollstein, and P. Hainaut, TP53 mutations in human cancers: origins, consequences, and clinical use. Cold Spring Harb Perspect Biol, 2010. 2(1): p. a001008.

8. Barta, J.A. and S.B. McMahon, Lung-Enriched Mutations in the $p 53$ Tumor Suppressor: $A$ Paradigm for Tissue-Specific Gain of Oncogenic Function. Mol Cancer Res, 2019. 17(1): p. 3-9.

9. Robles, A.I. and C.C. Harris, Clinical outcomes and correlates of TP53 mutations and cancer. Cold Spring Harb Perspect Biol, 2010. 2(3): p. a001016.

10. Brosh, R. and V. Rotter, When mutants gain new powers: news from the mutant p53 field. Nat Rev Cancer, 2009. 9(10): p. 701-13.

11. el-Deiry, W.S., et al., Definition of a consensus binding site for p53. Nat Genet, 1992. 1(1): p. 459.

12. Wang, W., S.H. Kim, and W.S. El-Deiry, Small-molecule modulators of p53 family signaling and antitumor effects in p53-deficient human colon tumor xenografts. Proc Natl Acad Sci U S A, 2006. 103(29): p. 11003-8.

13. Zhang, S., et al., Small-Molecule NSC59984 Restores p53 Pathway Signaling and Antitumor Effects against Colorectal Cancer via p73 Activation and Degradation of Mutant $p 53$. Cancer Res, 2015. 75(18): p. 3842-52.

14. Hong, B., et al., Prodigiosin rescues deficient $p 53$ signaling and antitumor effects via upregulating $p 73$ and disrupting its interaction with mutant $p 53$. Cancer Res, 2014. 74(4): p. 1153-65.

15. Richardson, C., et al., Small-molecule CB002 restores $p 53$ pathway signaling and represses colorectal cancer cell growth. Cell Cycle, 2017. 16(18): p. 1719-1725.

16. Hernandez-Borrero, L.J., et al., CB002, a novel p53 tumor suppressor pathway-restoring small molecule induces tumor cell death through the pro-apoptotic protein NOXA. Cell Cycle, 2018. 17(5): p. 557-567.

17. Schlichtholz, B., et al., Analyses of $p 53$ antibodies in sera of patients with lung carcinoma define immunodominant regions in the $p 53$ protein. Br J Cancer, 1994. 69(5): p. 809-16.

18. Schlichtholz, B., et al., The immune response to $p 53$ in breast cancer patients is directed against immunodominant epitopes unrelated to the mutational hot spot. Cancer Res, 1992. 52(22): $\mathrm{p}$. 6380-4.

19. Haupt, Y., et al., Mdm2 promotes the rapid degradation of p53. Nature, 1997. 387(6630): p. 2969.

20. Lain, S., et al., Discovery, in vivo activity, and mechanism of action of a small-molecule p53 activator. Cancer Cell, 2008. 13(5): p. 454-63.

21. Boeckler, F.M., et al., Targeted rescue of a destabilized mutant of $p 53$ by an in silico screened drug. Proc Natl Acad Sci U S A, 2008. 105(30): p. 10360-5. 
22. Demma, M., et al., SCH529074, a small molecule activator of mutant $p 53$, which binds $p 53$ DNA binding domain (DBD), restores growth-suppressive function to mutant $p 53$ and interrupts HDM2-mediated ubiquitination of wild type p53. J Biol Chem. 285(14): p. 10198-212.

23. Butler, J.S. and S.N. Loh, Structure, function, and aggregation of the zinc-free form of the p53 DNA binding domain. Biochemistry, 2003. 42(8): p. 2396-403.

24. Yu, X., et al., Allele-specific p53 mutant reactivation. Cancer Cell, 2012. 21(5): p. 614-625.

25. Galea, C., P. Bowman, and R.W. Kriwacki, Disruption of an intermonomer salt bridge in the $p 53$ tetramerization domain results in an increased propensity to form amyloid fibrils. Protein Sci, 2005. 14(12): p. 2993-3003.

26. Zawacka-Pankau, J. and G. Selivanova, Pharmacological reactivation of $p 53$ as a strategy to treat cancer. J Intern Med, 2015. 277(2): p. 248-259.

27. Lampreht Tratar, U., S. Horvat, and M. Cemazar, Transgenic Mouse Models in Cancer Research. Front Oncol, 2018. 8: p. 268.

28. Kersten, K., et al., Genetically engineered mouse models in oncology research and cancer medicine. EMBO Mol Med, 2017. 9(2): p. 137-153.

29. Kenzelmann Broz, D. and L.D. Attardi, In vivo analysis of p53 tumor suppressor function using genetically engineered mouse models. Carcinogenesis, 2010. 31(8): p. 1311-8.

30. Harvey, M., et al., Spontaneous and carcinogen-induced tumorigenesis in p53-deficient mice. Nat Genet, 1993. 5(3): p. 225-9.

31. Schwitalla, S., et al., Loss of p53 in enterocytes generates an inflammatory microenvironment enabling invasion and lymph node metastasis of carcinogen-induced colorectal tumors. Cancer Cell, 2013. 23(1): p. 93-106.

32. French, J.E., et al., Loss of heterozygosity frequency at the Trp53 locus in p53-deficient (+/-) mouse tumors is carcinogen-and tissue-dependent. Carcinogenesis, 2001. 22(1): p. 99-106.

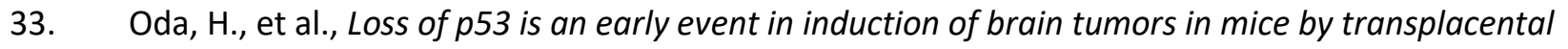
carcinogen exposure. Cancer Res, 1997. 57(4): p. 646-50.

34. Sakai, H., et al., High susceptibility of nullizygous p53 knockout mice to colorectal tumor induction by 1,2-dimethylhydrazine. J Cancer Res Clin Oncol, 2003. 129(6): p. 335-40.

35. Ozaki, K., et al., High susceptibility of p53(+/-) knockout mice in N-butyl-N-(4hydroxybutyl)nitrosamine urinary bladder carcinogenesis and lack of frequent mutation in residual allele. Cancer Res, 1998. 58(17): p. 3806-11.

36. Kado, S., et al., Intestinal microflora are necessary for development of spontaneous adenocarcinoma of the large intestine in T-cell receptor beta chain and p53 double-knockout mice. Cancer Res, 2001. 61(6): p. 2395-8.

37. Yamamoto, M., et al., $p 53$ knockout mice (-/-) are more susceptible than (+/-) or (+/+) mice to $\mathrm{N}$ methyl-N-nitrosourea stomach carcinogenesis. Carcinogenesis, 2000. 21(10): p. 1891-7.

38. Zhou, X., et al., Pharmacological activation of $p 53$ triggers viral mimicry response thereby abolishing tumor immune evasion and promoting anti-tumor immunity. Cancer Discov, 2021.

39. Blagih, J., et al., Cancer-Specific Loss of p53 Leads to a Modulation of Myeloid and T Cell Responses. Cell Rep, 2020. 30(2): p. 481-496 e6.

40. Christophorou, M.A., et al., Temporal dissection of $p 53$ function in vitro and in vivo. Nat Genet, 2005. 37(7): p. 718-26.

41. Martins, C.P., L. Brown-Swigart, and G.I. Evan, Modeling the therapeutic efficacy of p53 restoration in tumors. Cell, 2006. 127(7): p. 1323-34.

42. Hanel, W., et al., Two hot spot mutant p53 mouse models display differential gain of function in tumorigenesis. Cell Death Differ, 2013. 20(7): p. 898-909.

43. Lang, G.A., et al., Gain of function of a p53 hot spot mutation in a mouse model of Li-Fraumeni syndrome. Cell, 2004. 119(6): p. 861-72.

44. Olive, K.P., et al., Mutant $p 53$ gain of function in two mouse models of Li-Fraumeni syndrome. Cell, 2004. 119(6): p. 847-60. 
45. Alexandrova, E.M., et al., Improving survival by exploiting tumour dependence on stabilized mutant $p 53$ for treatment. Nature, 2015. 523(7560): p. 352-6.

46. Tchelebi, L., H. Ashamalla, and P.R. Graves, Mutant $p 53$ and the response to chemotherapy and radiation. Subcell Biochem, 2014. 85: p. 133-59.

47. Chen, J., The Cell-Cycle Arrest and Apoptotic Functions of p53 in Tumor Initiation and Progression. Cold Spring Harb Perspect Med, 2016. 6(3): p. a026104.

48. Vega-Stromberg, T., Chemotherapy-induced Secondary Malignancies. Journal of Infusion Nursing, 2003. 26(6): p. 353-361.

49. Li, H., et al., Targeting the Oncogenic p53 Mutants in Colorectal Cancer and Other Solid Tumors. Int J Mol Sci, 2019. 20(23).

50. Zhu, G., et al., Mutant p53 in Cancer Progression and Targeted Therapies. Front Oncol, 2020. 10: p. 595187.

51. Duffy, M.J., et al., Targeting p53 for the treatment of cancer. Semin Cancer Biol, 2020.

52. Bykov, V.J.N., et al., Targeting mutant p53 for efficient cancer therapy. Nat Rev Cancer, 2018. 18(2): p. 89-102.

53. Duffy, M.J., N.C. Synnott, and J. Crown, Mutant $p 53$ as a target for cancer treatment. European Journal of Cancer, 2017. 83: p. 258-265.

54. Sabapathy, K. and D.P. Lane, Therapeutic targeting of p53: all mutants are equal, but some mutants are more equal than others. Nature Reviews Clinical Oncology, 2018. 15(1): p. 13-30.

55. Perdrix, A., et al., PRIMA-1 and PRIMA-1Met (APR-246): From Mutant/Wild Type p53 Reactivation to Unexpected Mechanisms Underlying Their Potent Anti-Tumor Effect in Combinatorial Therapies. Cancers, 2017. 9(12): p. 172.

56. Issaeva, N., et al., Small molecule RITA binds to $p 53$, blocks p53-HDM-2 interaction and activates p53 function in tumors. Nat Med, 2004. 10(12): p. 1321-8.

57. Sanz, G., et al., Inhibition of p53 inhibitors: progress, challenges and perspectives. J Mol Cell Biol, 2019. 11(7): p. 586-599.

58. Wurz, R.P. and V.J. Cee, Targeted Degradation of MDM2 as a New Approach to Improve the Efficacy of MDM2-p53 Inhibitors. J Med Chem, 2019. 62(2): p. 445-447.

59. Levine, A.J., Targeting Therapies for the $p 53$ Protein in Cancer Treatments. Annual Review of Cancer Biology, 2019. 3(1): p. 21-34.

60. Heise, C., et al., ONYX-015, an E1B gene-attenuated adenovirus, causes tumor-specific cytolysis and antitumoral efficacy that can be augmented by standard chemotherapeutic agents. Nat Med, 1997. 3(6): p. 639-45.

61. Zhang, W.W., et al., The First Approved Gene Therapy Product for Cancer Ad-p53 (Gendicine): 12 Years in the Clinic. Hum Gene Ther, 2018. 29(2): p. 160-179.

62. Pearson, S., H. Jia, and K. Kandachi, China approves first gene therapy. Nat Biotechnol, 2004. 22(1): p. 3-4.

63. Foster, B.A., et al., Pharmacological rescue of mutant p53 conformation and function. Science, 1999. 286(5449): p. 2507-10.

64. Bykov, V.J., et al., Restoration of the tumor suppressor function to mutant $p 53$ by a lowmolecular-weight compound. Nat Med, 2002. 8(3): p. 282-8.

65. Duffy, M.J., et al., Targeting p53 for the treatment of cancer. Seminars in Cancer Biology, 2020.

66. Bykov, V.J., et al., PRIMA-1(MET) synergizes with cisplatin to induce tumor cell apoptosis. Oncogene, 2005. 24(21): p. 3484-91.

67. Bykov, V.J., et al., Reactivation of mutant p53 and induction of apoptosis in human tumor cells by maleimide analogs. J Biol Chem, 2005. 280(34): p. 30384-91.

68. Zache, N., et al., Mutant p53 targeting by the low molecular weight compound STIMA-1. Mol Oncol, 2008. 2(1): p. 70-80.

69. Kaar, J.L., et al., Stabilization of mutant p53 via alkylation of cysteines and effects on DNA binding. Protein Sci, 2010. 19(12): p. 2267-78. 
70. Punganuru, S.R., et al., Design and synthesis of a C7-aryl piperlongumine derivative with potent antimicrotubule and mutant p53-reactivating properties. Eur J Med Chem, 2016. 107: p. 233-44.

71. Bauer, M.R., A.C. Joerger, and A.R. Fersht, 2-Sulfonylpyrimidines: Mild alkylating agents with anticancer activity toward p53-compromised cells. Proc Natl Acad Sci U S A, 2016. 113(36): p. E5271-80.

72. Synnott, N.C., et al., COTI-2 reactivates mutant $p 53$ and inhibits growth of triple-negative breast cancer cells. Breast Cancer Res Treat, 2020. 179(1): p. 47-56.

73. Tal, P., et al., Cancer therapeutic approach based on conformational stabilization of mutant p53 protein by small peptides. Oncotarget, 2016. 7(11): p. 11817-37.

74. Proia, D.A. and R.C. Bates, Ganetespib and HSP9O: translating preclinical hypotheses into clinical promise. Cancer Res, 2014. 74(5): p. 1294-300.

75. Li, D., N.D. Marchenko, and U.M. Moll, SAHA shows preferential cytotoxicity in mutant $p 53$ cancer cells by destabilizing mutant $p 53$ through inhibition of the HDAC6-Hsp90 chaperone axis. Cell Death Differ, 2011. 18(12): p. 1904-13.

76. Foggetti, G., et al., Autophagy induced by SAHA affects mutant P53 degradation and cancer cell survival. Biosci Rep, 2019. 39(2).

77. Parrales, A., et al., DNAJA1 controls the fate of misfolded mutant $p 53$ through the mevalonate pathway. Nat Cell Biol, 2016. 18(11): p. 1233-1243.

78. Vakifahmetoglu-Norberg, H., et al., Chaperone-mediated autophagy degrades mutant $p 53$. Genes Dev, 2013. 27(15): p. 1718-30.

79. Kravchenko, J.E., et al., Small-molecule RETRA suppresses mutant p53-bearing cancer cells through a p73-dependent salvage pathway. Proc Natl Acad Sci U S A, 2008. 105(17): p. 6302-7.

80. Hsiue, E.H., et al., Targeting a neoantigen derived from a common TP53 mutation. Science, 2021. 371(6533).

81. Grinkevich, V.V., et al., Ablation of key oncogenic pathways by RITA-reactivated p53 is required for efficient apoptosis. Cancer Cell, 2009. 15(5): p. 441-53.

82. Ahmed, A., et al., Pharmacological activation of a novel p53-dependent S-phase checkpoint involving CHK-1. Cell Death Dis. 2: p. e160.

83. Yang, J., et al., Small-molecule activation of $p 53$ blocks hypoxia-inducible factor 1alpha and vascular endothelial growth factor expression in vivo and leads to tumor cell apoptosis in normoxia and hypoxia. Mol Cell Biol, 2009. 29(8): p. 2243-53.

84. Shi, Y., et al., ROS-dependent activation of JNK converts $p 53$ into an efficient inhibitor of oncogenes leading to robust apoptosis. Cell Death Differ, 2014. 21(4): p. 612-23.

85. Wiegering, A., et al., Reactivating $p 53$ and Inducing Tumor Apoptosis (RITA) Enhances the Response of RITA-Sensitive Colorectal Cancer Cells to Chemotherapeutic Agents 5-Fluorouracil and Oxaliplatin. Neoplasia, 2017. 19(4): p. 301-309.

86. Weilbacher, A., et al., RITA can induce cell death in p53-defective cells independently of p53 function via activation of JNK/SAPK and p38. Cell Death Dis, 2014. 5: p. e1318.

87. Hu, C., et al., Design, synthesis, and biological evaluation of imidazoline derivatives as $p 53-$ MDM2 binding inhibitors. Bioorg Med Chem. 19(18): p. 5454-61.

88. Ray-Coquard, I., et al., Effect of the MDM2 antagonist RG7112 on the P53 pathway in patients with MDM2-amplified, well-differentiated or dedifferentiated liposarcoma: an exploratory proofof-mechanism study. Lancet Oncol, 2012. 13(11): p. 1133-40.

89. Ding, Q., et al., Discovery of RG7388, a potent and selective p53-MDM2 inhibitor in clinical development. J Med Chem, 2013. 56(14): p. 5979-83.

90. Verreault, M., et al., Preclinical Efficacy of the MDM2 Inhibitor RG7112 in MDM2-Amplified and TP53 Wild-type Glioblastomas. Clin Cancer Res, 2016. 22(5): p. 1185-96.

91. Andreeff, M., et al., Results of the Phase I Trial of RG7112, a Small-Molecule MDM2 Antagonist in Leukemia. Clin Cancer Res, 2016. 22(4): p. 868-76.

92. Patnaik, A., et al., Clinical pharmacology characterization of RG7112, an MDM2 antagonist, in patients with advanced solid tumors. Cancer Chemother Pharmacol, 2015. 76(3): p. 587-95. 
93. Her, N.G., et al., Potent effect of the MDM2 inhibitor AMG232 on suppression of glioblastoma stem cells. Cell Death Dis, 2018. 9(8): p. 792.

94. Shadfan, M., V. Lopez-Pajares, and Z.M. Yuan, MDM2 and MDMX: Alone and together in regulation of p53. Transl Cancer Res, 2012. 1(2): p. 88-89.

95. Reed, D., et al., Identification and characterization of the first small molecule inhibitor of MDMX. J Biol Chem, 2010. 285(14): p. 10786-96.

96. Carvajal, L.A., et al., Dual inhibition of MDMX and MDM2 as a therapeutic strategy in leukemia. Sci Transl Med, 2018. 10(436).

97. Saleh, M.N., et al., Phase 1 Trial of ALRN-6924, a Dual Inhibitor of MDMX and MDM2, in Patients with Solid Tumors and Lymphomas Bearing Wild-Type TP53. Clin Cancer Res, 2021.

98. Gasparian, A.V., et al., Curaxins: anticancer compounds that simultaneously suppress NF-kappaB and activate $p 53$ by targeting FACT. Sci Transl Med, 2011. 3(95): p. 95 ra74.

99. Bae, S.K., et al., Induction of apoptosis in colon cancer cells by a novel topoisomerase I inhibitor Topln. Biochem Biophys Res Commun, 2011. 409(1): p. 75-81.

100. Lambert, J.M., et al., PRIMA-1 reactivates mutant $p 53$ by covalent binding to the core domain. Cancer Cell, 2009. 15(5): p. 376-88.

101. Zhang, Q., et al., APR-246 reactivates mutant $p 53$ by targeting cysteines 124 and 277 . Cell Death Dis, 2018. 9(5): p. 439.

102. Demma, M., et al., SCH529074, a small molecule activator of mutant $p 53$, which binds $p 53$ DNA binding domain (DBD), restores growth-suppressive function to mutant $p 53$ and interrupts HDM2-mediated ubiquitination of wild type p53. J Biol Chem, 2010. 285(14): p. 10198-212.

103. Lin, K., et al., Hsp90 inhibition has opposing effects on wild-type and mutant $p 53$ and induces p21 expression and cytotoxicity irrespective of p53/ATM status in chronic lymphocytic leukaemia cells. Oncogene, 2008. 27(17): p. 2445-55.

104. Li, D., et al., Functional inactivation of endogenous MDM2 and CHIP by HSP9O causes aberrant stabilization of mutant $p 53$ in human cancer cells. Mol Cancer Res, 2011. 9(5): p. 577-88.

105. Peng, Y., et al., Inhibition of MDM2 by hsp90 contributes to mutant $p 53$ stabilization. J Biol Chem, 2001. 276(44): p. 40583-90.

106. Hu, Z., et al., Full-Length Transcriptome Assembly of Italian Ryegrass Root Integrated with RNASeq to Identify Genes in Response to Plant Cadmium Stress. Int J Mol Sci, 2020. 21(3).

107. Qi, Z., et al., Design and linkage optimization of ursane-thalidomide-based PROTACs and identification of their targeted-degradation properties to MDM2 protein. Bioorg Chem, 2021. 111: p. 104901.

108. Galbraith, M.D., et al., Global Analyses to Identify Direct Transcriptional Targets of p53. Methods Mol Biol, 2021. 2267: p. 19-56.

109. Allen, M.A., et al., Global analysis of p53-regulated transcription identifies its direct targets and unexpected regulatory mechanisms. Elife, 2014. 3: p. e02200.

110. Kenzelmann Broz, D., et al., Global genomic profiling reveals an extensive p53-regulated autophagy program contributing to key 553 responses. Genes Dev, 2013. 27(9): p. 1016-31.

111. Menendez, D., et al., Diverse stresses dramatically alter genome-wide $p 53$ binding and transactivation landscape in human cancer cells. Nucleic Acids Res, 2013. 41(15): p. 7286-301.

112. Fischer, M., Census and evaluation of p53 target genes. Oncogene, 2017. 36(28): p. 3943-3956.

113. Li, M., The role of P53 up-regulated modulator of apoptosis (PUMA) in ovarian development, cardiovascular and neurodegenerative diseases. Apoptosis, 2021. 26(5-6): p. 235-247.

114. Sharma, K., et al., p53-independent Noxa induction by cisplatin is regulated by ATF3/ATF4 in head and neck squamous cell carcinoma cells. Mol Oncol, 2018. 12(6): p. 788-798.

115. Armstrong, J.L., et al., Role of Noxa in p53-independent fenretinide-induced apoptosis of neuroectodermal tumours. Apoptosis, 2007. 12(3): p. 613-22.

116. Perez-Galan, P., et al., The proteasome inhibitor bortezomib induces apoptosis in mantle-cell lymphoma through generation of ROS and Noxa activation independent of p53 status. Blood, 2006. 107(1): p. 257-64. 
117. Warfel, N.A. and W.S. El-Deiry, p21WAF1 and tumourigenesis: 20 years after. Curr Opin Oncol, 2013. 25(1): p. 52-8.

118. Tian, X., et al., P53-independent partial restoration of the $p 53$ pathway in tumors with mutated p53 through ATF4 transcriptional modulation by ERK1/2 and CDK9. Neoplasia, 2021. 23(3): p. 304-325.

119. Hernandez Borrero, L., et al., A subset of CBO02 xanthine analogs bypass p53-signaling to restore a p53 transcriptome and target an S-phase cell cycle checkpoint in tumors with mutated-p53. Elife, 2021. 10.

120. DeLeo, A.B. and E. Appella, The p53 Saga: Early Steps in the Development of Tumor Immunotherapy. J Immunol, 2020. 204(9): p. 2321-2328.

121. Kunimura, N., et al., Combination of rAd-p53 in situ gene therapy and anti-PD-1 antibody immunotherapy induced anti-tumor activity in mouse syngeneic urogenital cancer models. Sci Rep, 2020. 10(1): p. 17464.

122. Hardwick, N.R., et al., p53MVA therapy in patients with refractory gastrointestinal malignancies elevates p53-specific CD8+ T-cell responses. Clin Cancer Res, 2014. 20(17): p. 4459-70.

123. Chung, V., et al., Evaluation of safety and efficacy of $553 M V A$ vaccine combined with pembrolizumab in patients with advanced solid cancers. Clin Transl Oncol, 2019. 21(3): p. 363372.

124. Hao, Q., H. Lu, and X. Zhou, A potential synthetic lethal strategy with PARP inhibitors: perspective on 'Inactivation of the tumor suppressor $p 53$ by long noncoding RNA RMRP'. J Mol Cell Biol, 2021.

125. Yap, T.A., A. Omlin, and J.S. de Bono, Development of therapeutic combinations targeting major cancer signaling pathways. J Clin Oncol, 2013. 31(12): p. 1592-605.

126. Grellety, T., et al., PRIMA-1(MET) induces death in soft-tissue sarcomas cell independent of $p 53$. BMC Cancer, 2015. 15: p. 684.

127. Fujihara, K.M., et al., SLC7A11 is a superior determinant of APR-246 (Eprenetapopt) response than TP53 mutation status. Mol Cancer Ther, 2021.

128. Liu, D.S., et al., Inhibiting the system $\times C(-) /$ glutathione axis selectively targets cancers with mutant-p53 accumulation. Nat Commun, 2017. 8: p. 14844.

129. Li, Y., et al., Selective induction of apoptosis in mutant $p 53$ premalignant and malignant cancer cells by PRIMA-1 through the c-Jun-NH2-kinase pathway. Mol Cancer Ther, 2005. 4(6): p. 901-9.

130. Saha, M.N., et al., Targeting p53 via JNK pathway: a novel role of RITA for apoptotic signaling in multiple myeloma. PLoS One, 2012. 7(1): p. e30215.

131. Roy, S., et al., MDM2 Antagonists Induce a Paradoxical Activation of Erk1/2 through a P53Dependent Mechanism in Dedifferentiated Liposarcomas: Implications for Combinatorial Strategies. Cancers (Basel), 2020. 12(8).

132. Ji, Z., et al., Vemurafenib synergizes with nutlin-3 to deplete survivin and suppresses melanoma viability and tumor growth. Clin Cancer Res, 2013. 19(16): p. 4383-91.

133. Vatsyayan, R., et al., Nutlin-3 enhances sorafenib efficacy in renal cell carcinoma. Mol Carcinog, 2013. 52(1): p. 39-48.

134. Aubrey, B.J., et al., How does $p 53$ induce apoptosis and how does this relate to $p 53-m e d i a t e d$ tumour suppression? Cell Death Differ, 2018. 25(1): p. 104-113.

135. Bragado, P., et al., Apoptosis by cisplatin requires $p 53$ mediated p38alpha MAPK activation through ROS generation. Apoptosis, 2007. 12(9): p. 1733-42.

136. Grandela, C., et al., $p 53$ is required for etoposide-induced apoptosis of human embryonic stem cells. Stem Cell Res, 2007. 1(2): p. 116-28.

137. Sun, Y., et al., P53 is required for Doxorubicin-induced apoptosis via the TGF-beta signaling pathway in osteosarcoma-derived cells. Am J Cancer Res, 2016. 6(1): p. 114-25.

138. Saha, M.N., et al., Small molecule MIRA-1 induces in vitro and in vivo anti-myeloma activity and synergizes with current anti-myeloma agents. Br J Cancer, 2014. 110(9): p. 2224-31. 
139. Xie, Q., et al., Synergistic anticancer effect of exogenous wild-type p53 gene combined with 5-FU in human colon cancer resistant to 5-FU in vivo. World J Gastroenterol, 2016. 22(32): p. 7342-52.

140. Pairawan, S., et al., First in class dual MDM2/MDMX inhibitor ALRN-6924 enhances antitumor efficacy of chemotherapy in TP53 wild-type hormone receptor-positive breast cancer models. Breast Cancer Res, 2021. 23(1): p. 29.

141. Duffy, M.J., N.C. Synnott, and J. Crown, Mutant p53 as a target for cancer treatment. Eur J Cancer, 2017. 83: p. 258-265.

142. Magrini, R., et al., PRIMA-1 synergizes with adriamycin to induce cell death in non-small cell lung cancer cells. J Cell Biochem, 2008. 104(6): p. 2363-73.

143. Messina, R.L., et al., Reactivation of $p 53$ mutants by prima-1 [corrected] in thyroid cancer cells. Int J Cancer, 2012. 130(10): p. 2259-70.

144. Mohell, N., et al., APR-246 overcomes resistance to cisplatin and doxorubicin in ovarian cancer cells. Cell Death Dis, 2015. 6: p. e1794.

145. Lin, A.B., S.C. McNeely, and R.P. Beckmann, Achieving Precision Death with Cell-Cycle Inhibitors that Target DNA Replication and Repair. Clin Cancer Res, 2017. 23(13): p. 3232-3240.

146. Zhang, Y. and T. Hunter, Roles of Chk1 in cell biology and cancer therapy. Int J Cancer, 2014. 134(5): p. 1013-23.

147. Gadhikar, M.A., et al., Chk1/2 inhibition overcomes the cisplatin resistance of head and neck cancer cells secondary to the loss of functional p53. Mol Cancer Ther, 2013. 12(9): p. 1860-73.

148. Ma, C.X., et al., Targeting Chk1 in p53-deficient triple-negative breast cancer is therapeutically beneficial in human-in-mouse tumor models. J Clin Invest, 2012. 122(4): p. 1541-52.

149. Vance, S., et al., Selective radiosensitization of p53 mutant pancreatic cancer cells by combined inhibition of Chk1 and PARP1. Cell Cycle, 2011. 10(24): p. 4321-9.

150. Geenen, J.J.J. and J.H.M. Schellens, Molecular Pathways: Targeting the Protein Kinase Wee1 in Cancer. Clin Cancer Res, 2017. 23(16): p. 4540-4544.

151. Yin, Y., et al., Wee1 inhibition can suppress tumor proliferation and sensitize $p 53$ mutant colonic cancer cells to the anticancer effect of irinotecan. Mol Med Rep, 2018. 17(2): p. 3344-3349.

152. Rajeshkumar, N.V., et al., MK-1775, a potent Wee1 inhibitor, synergizes with gemcitabine to achieve tumor regressions, selectively in p53-deficient pancreatic cancer xenografts. Clin Cancer Res, 2011. 17(9): p. 2799-806.

153. Meng, X., et al., AZD1775 Increases Sensitivity to Olaparib and Gemcitabine in Cancer Cells with p53 Mutations. Cancers (Basel), 2018. 10(5).

154. Oza, A.M., et al., A Biomarker-enriched, Randomized Phase II Trial of Adavosertib (AZD1775) Plus Paclitaxel and Carboplatin for Women with Platinum-sensitive TP53-mutant Ovarian Cancer. Clin Cancer Res, 2020. 26(18): p. 4767-4776.

155. Leijen, S., et al., Phase II Study of WEE1 Inhibitor AZD1775 Plus Carboplatin in Patients With TP53-Mutated Ovarian Cancer Refractory or Resistant to First-Line Therapy Within 3 Months. J Clin Oncol, 2016. 34(36): p. 4354-4361.

156. Gorecki, L., M. Andrs, and J. Korabecny, Clinical Candidates Targeting the ATR-CHK1-WEE1 Axis in Cancer. Cancers (Basel), 2021. 13(4).

157. Kong, A. and H. Mehanna, WEE1 Inhibitor: Clinical Development. Curr Oncol Rep, 2021. 23(9): p. 107.

158. Sobol, R.E., et al., Analysis of Adenoviral p53 Gene Therapy Clinical Trials in Recurrent Head and Neck Squamous Cell Carcinoma. Front Oncol, 2021. 11: p. 645745.

159. Moore, E.C., et al., Nanocomplex-based TP53 gene therapy promotes anti-tumor immunity through TP53- and STING-dependent mechanisms. Oncoimmunology, 2018. 7(7): p. e1404216.

160. Batir, M.B., E. Sahin, and F.S. Cam, Evaluation of the CRISPR/Cas9 directed mutant TP53 gene repairing effect in human prostate cancer cell line PC-3. Mol Biol Rep, 2019. 46(6): p. 6471-6484.

161. Chira, S., et al., Restoring the $p 53$ 'Guardian' Phenotype in p53-Deficient Tumor Cells with CRISPR/Cas9. Trends Biotechnol, 2018. 36(7): p. 653-660. 
162. Mirgayazova, R., et al., Therapeutic Editing of the TP53 Gene: Is CRISPR/Cas9 an Option? Genes (Basel), 2020. 11(6). 